\title{
Analysis of Arrhenius Kinetics on Multiphase Flow between a Pair of Rotating Circular Plates
}

\author{
M. B. Arain, ${ }^{1}$ M. M. Bhatti $\mathbb{D}^{,},{ }^{2}$ A. Zeeshan, ${ }^{1}$ Tareq Saeed, ${ }^{3}$ and Aatef Hobiny ${ }^{3}$ \\ ${ }^{1}$ Department of Mathematics and Statistics, International Islamic University, Islamabad 44000, Pakistan \\ ${ }^{2}$ College of Mathematics and Systems Science, Shandong University of Science and Technology, Qingdao, \\ Shandong 266590, China \\ ${ }^{3}$ Nonlinear Analysis and Applied Mathematics (NAAM)-Research Group, Department of Mathematics, Faculty of Science, \\ King Abdulaziz University, P.O. Box 80203, Jeddah 21589, Saudi Arabia
}

Correspondence should be addressed to M. M. Bhatti; mmbhatti@sdust.edu.cn

Received 5 August 2020; Revised 5 November 2020; Accepted 11 November 2020; Published 1 December 2020

Academic Editor: Bekir Sahin

Copyright (c) 2020 M. B. Arain et al. This is an open access article distributed under the Creative Commons Attribution License, which permits unrestricted use, distribution, and reproduction in any medium, provided the original work is properly cited.

In this study, we aim to deal with the flow behavior betwixt a pair of rotating circular plates filled with Carreau fluid under the suspension of nanoparticles and motile gyrotactic microorganisms in the presence of generalized magnetic Reynolds number. The activation energy is also contemplated with the nanoparticle concentration equation. The appropriate similarity transformations are used to formulate the proposed mathematical modeling in the three dimensions. The outcomes of the torque on both plates, i.e., the fix and the moving plate, are also contemplated. A well-known differential transform method (DTM) with a combination of Padé approximation will be implemented to get solutions to the coupled nonlinear ordinary differential equations (ODEs). The impact of different nondimensional physical aspects on velocity profile, temperature, concentration, and motile gyrotactic microorganism functions is discussed. The shear-thinning fluid viscosity decreases with shear strain due to its high velocity compared to the Newtonian and shear-thickening case. The impact of Carreau fluid velocity for shear-thinning $(n<1)$, Newtonian case $(n=0)$, and shear-thickening $(n>1)$ cases on axial velocity distribution $f^{\prime}(\lambda)$ has been discussed in tabular form and graphical figures. For the validation of the current methodology, a comparison is made between DTM-Padé and the numerical shooting scheme.

\section{Introduction}

A nanofluid is a substance that contains nanosized particles suspended in the base fluid. These nanoparticles are usually made of oxides, metals, carbon nanotubes, or carbides, while base fluids are often taken as water, oil, and ethylene glycol. The potential use of nanofluids can be found in many applications in industry, i.e., heat transfer, especially in pharmaceutical processes, microelectronics, engine cooling (vehicle heat management), hybrid-power engines, chiller, fuel cells, heat exchanger, grinding, and boiler flue gas temperature reduction. The non-Newtonian fluids have significant applications in science and engineering, such as coating of wire and blade, textile dying, papers, manufacturing of plastics, the flow of biological liquids, and food processing. In industrial applications, the flow of non-
Newtonian viscoelastic Carreau nanofluids is essential to increase energy performance. For example, it is used to release polymer sheets from the die or in plastic film drawing. Non-Newtonian fluids have drawn considerable interest among researchers and scientists due to their broad applications. Examples include apple sauce, chyme, photographic emulsion, dirt, soaps, blood, and shampoos at low shear stress that may be noted as non-Newtonian fluids. In the chemical engineering industry, the viscosity of fluid plays a significant role. Viscosity is dependent on the shear rate in the case of generalized Newtonian fluids. The idea of the generalized non-Newtonian fluid was introduced by Bird et al. [1]. Fluid flow past a rigid surface has also been investigated, and literature suggests that the microlevel surface forces are essential and that fluid layering increases viscosity. They help to increase the viscosity of fluid due to fluid 
coating. In the power-law model, the limitation is that when the shear rate is extremely low or high, the viscosity is not adequately addressed. To tackle this problem, the model of the Carreau fluid model is presented [2]. The Carreau fluid model gains the attention of many researchers for several years because of their essential characteristics. The flow on the magnetized permeable shrinking sheet and radiation due to heat was discussed by Yahya et al. [3]. Eid et al. [4] analyzed flow over a nonlinear permeable stretching sheet due to Carreau fluid under the influence of chemical reaction. Santoshi et al. [5] numerically studied the Carreau nanofluids in three dimensions on a stretching sheet in addition to considering mass slip and nonlinear thermal radiation. The effects of the internal energy in the porous von Karman model in steady electrical Carreau fluid under ohmic heating and transverse magnetohydrodynamics were studied by Khan et al. [6]. They considered a cylindrical coordinate system, and similarity transformations are applied to obtain the ordinary differential equation for the proposed problem and implement a well-known shooting scheme to achieve the results. Bilal et al. [7] investigated flow features of Carreau fluid by conferring the stimulating traits of thermal stratification. Appropriate use of MHD and infinite shear rate viscosity flow equation are modeled. They concluded that the thermal stratification characterizes fluid flow's thermal distribution, and Carreau fluid acts in a reverse direction for shearthickening and shear-thinning liquids. Khan et al. [8] examined the mass and heat transfer for convection in nonNewtonian Carreau nanofluid on a cylinder in the presence of temperature-dependent thermal conductivity. They considered a well-known model, i.e., Buongiorno's model, which contains the Brownian and thermophoresis parameters. The key finding of this study was that the temperature profile and concentration of nanoparticle were increasing functions for the thermophoresis parameter in shear-thickening and shearthinning fluids. Some recent critical studies related to the current topic are given in [9-13].

In previous decades, the study of magnet fields in fluid flow grabbed substantial attention because magnetohydrodynamics (MHD) is frequently used in many areas such as crystal growth process, pumping, agriculture, and polymer industry. MHD was recently identified as very useful in biotechnology as it is used in multiple testing processes for diseases. Recently, Lu et al. [14] studied mathematical models for the axisymmetric steady magnetohydrodynamic flow of Carreau nanofluids across radially stretched surfaces under nonlinear radiation of heat and chemical reaction. The additional feature of the problem, which makes it unique, is the generation/absorption of heat connected with new applied zero mass flux conditions. The flow due to boundary conditions due to convection with Carreau nanofluid with a magnetic field is studied by Wakif et al. [15] in addition to jump and slip conditions on a stretching cylinder. Khan et al. $[16,17]$ examined the flow induced by non-Newtonian Carreau fluid on a stretching cylinder with a magnetic field.

Further, the flow on a stretching cylinder affected by homogenous and heterogeneous conditions was examined by them and applied to convective boundary conditions numerically. This study mainly aims at the direct influence of homogenous and different reactions of Carreau fluid on a stretching cylinder with a magnetic field. A practical method for two-dimensional Carreau nanofluid for a nonsimilar solution with a magnetic field (applied) and mixed convection was analyzed by Sardar et al. [18]. They showed that increasing the buoyancy parameter boosts both skin friction and Nusselt number. In the presence of infinite shear rate viscosity, the stagnation point and the MHD flow of a Carreau fluid are also detected. Salahuddin et al. [19] examined the generalized slip effects of the magnetic field on Carreau nanofluid for a linear stretching cylinder with reactive species. Bhatti et al. [20] investigated the peristaltic motion of small particles suspended in a Carreau nanofluid with constant density. Laminar flow in two-dimensional past a stretching cylinder covered with the porous surface was studied with the effects of the magnetic field by Bovand et al. [21]. This article presented the findings of a numerical study of the circulatory cylindrical fluid flow under the influence of magnetohydrodynamics. Another numerical analysis is made by Amanulla et al. [15]. They studied the two-dimensional steady convective boundary layer flow over the surface of an isothermal sphere with the radial magnetic field and slip conditions. They perceived that growth in momentum slip parameters allows the skin friction coefficient to be reduced. In contrast, the local Nusselt number declined as the Carreau fluid parameter increases. Rudraswamy et al. [22] examined the Carreau fluid flow in a two-dimensional stretchable surface with the magnetic field. They concluded with a statement that when Brownian motion parameter and thermophoretic parameters are enhanced, the temperature of the fluid increases. Kumar et al. [23] investigated the Cattaneo-Christov heat flux model with Carreau fluid under the magnetic field on a varying thickness of the melting surface. Akbar et al. [24] investigated the flow of stagnation point for Carreau fluid on a wrinkled sheet in two dimensions. They presented a dual solution for the problem under consideration, and the graphs for various parameters in the equations are drawn. Shit et al. [25] investigated the nanofluid flow with MHD and thermal radiation. They studied the system of energy efficiency through the Bejan number.

Due to the complicated nature of the chemical reactions system, restricting to binary type alone becomes more comfortable and straightforward. A chemical reaction requires a quantity of activation energy to start. In the Arrhenius equation, a reaction's activation energy is obtained by describing constant rate changes with the temperature. A chemical reaction is a chemical change in which reactants with varying properties may yield one or more products. Several industries need chemical reactions as an essential phase in the manufacturing process. Traditionally these types of responses occur in chemical reactors and are generally constrained to mass transfer. The response is sufficient when reagent volume and energy inputs enhance, and waste is reduced; then the optimum product can be obtained. Bestman [26] applied the model of binary reaction in the Arrhenius equation to produce a chemical reaction. The effects of energy of activation and binary chemical 
change on a two-dimensional radiative magnetohydrodynamics boundary layer flow for nanofluid on a vertical plate were discussed by Anuradha and Yegammai [27]. They scrutinized that the temperature distribution was accelerated, and the nanoparticle concentration profile decelerates under the effects of the heat generation, viscous dissipation, and MHD. Irfan et al. [28] discussed the Carreau nanofluid time-dependent flow for Arrhenius activation energy by using properties of binary chemical reaction with mixed convection. Khan et al. [29] explored the incompressible flow past a stretchable sheet of the Carreau-Yasuda model. Kumar et al. [30] analyzed the effects of nonlinear radiation with heat transfer attributes and activation energy. Khan et al. [29] reported a new nanofluid relation which examines the characteristics of the energy of activation with mixed convection Carreau nanofluid. They studied radiation and magnetic field parameters on both the entropy generation and the Bejan number.

Bioconvection flow is the flow of macroscopic convection of the fluid due to the density gradient generated by the collective swimming of motile microorganisms. Due to the swimming of these motile microorganisms in a particular direction, the density of the fluid increases, which results in the phenomenon of bioconvection. Slip effects and the movement of motile gyrotactic microorganisms on an exponentially stretching sheet were examined by Nayak at el. [31]. They discussed the temperature profile, velocity profile, nanoparticle concentration profile, and gyrotactic microorganism profile associated with different physical parameters. The thermal radiation effects with activation energy over a stretching cylinder with Oldroyd-B fluid due to the flow of microorganisms under the impact of the magnetic field were examined by Tlili et al. [32]. The thermal developmental nanofluid flow propagating on an extended surface was analyzed by Abdelmalek et al. [33] with additional activation energy characteristics, second-order, and viscous dissipation slip. Atif et al. [34] inspected the flow of the stratified micropolar MHD fluid with nanoparticles that contains gyrotactic microorganisms. The process of ohmic heating and radiated heat has also been taken into consideration. Khan et al. [35] determined the suspension of nanoparticles in Sisko nanofluid, utilizing the idea of microorganisms with activation energy and chemical reaction. Bhatti et al. [36] demonstrated the movement of motile gyrotactic microorganisms past a stretched surface filled with nanofluid. They applied the Successive Local Linearization Method (SLLM) for solving the modeled formulation and showed that the current method is stable and gives excellent results compared with similar methods. Nagendramma et al. [37] developed a mathematical model for Casson fluid through a slandering sheet in the existence of microorganisms for Cattaneo-Christov thermal flux and nonuniform heat source/sink. References [38-42] depict the flow in different geometries with different fluid models.

After investigating the above literature, it is found that no efforts are devoted to determining the analysis of activation energy for the Carreau non-Newtonian nanofluid with gyrotactic microorganism through a pair of rotating circular disks with generalized magnetic Reynolds number.
In the present formulation, we considered the induced magnetic field in the axial and tangential direction. The Carreau nanofluid with gyrotactic microorganisms is suspended betwixt the set of rotating circular disks. The effects of activation energy are also contemplated with nanoparticle concentration. A well-known differential transform method (DTM) with Padé approximant is employed to obtain the solutions of the given coupled highly nonlinear ordinary differential equations. The effects of all the parameters associated with velocity profile, temperature profile, induced magnetic field, nanoparticle concentration, and motile microorganism functions are discussed in detail via graphs and tables.

\section{Mathematical and Physical Modeling}

Let us contemplate the flow in three-dimensional axisymmetry in a squeezed lubricant film of a Carreau nanofluid betwixt a set of two circular rotating parallel plates with finite length. The coordinate system is chosen as cylindrical polar axis $(r, \theta, z)$ with the associated velocity field $V=\left[v_{r}, v_{\theta}, v_{z}\right]$. The heights of two parallel rotating circular plates are considered and taken as $\widehat{\Gamma}(t)\left[=(-\alpha t+1)^{1 / 2} D\right]$ at time $t$. The lower disk is stationary and upper disk is moving towards fix disk. The velocity of upper disk is denoted by $\widehat{\Gamma}^{\prime}(t)$. The $z$-axis indicates the symmetry axis in which the plates are rotating. The external magnetic field on the moving plate is applied with axial and azimuthal components, i.e.,

$$
\left\{\begin{array}{l}
\widehat{H}_{\theta}=\frac{r N_{0}}{\mu_{2}} \sqrt{\frac{D}{\widehat{\Gamma}(t)}}, \\
\widehat{H}_{z}=-\frac{\alpha M_{0} D}{\mu_{1} \widehat{\Gamma}(t)},
\end{array}\right.
$$

in which $M_{0}, N_{0}$ are the dimensionless quantities which make $\widehat{H}_{\theta}, \widehat{H}_{z}$ dimensionless and $\mu_{1}, \mu_{2}$ are the magnetic permeability's of the squeeze film and the medium external to the disks, respectively. For the liquid metals, $\mu_{2}=\mu_{\ell}$ where $\mu_{\ell}$ denotes the permeability of free space. $H_{\theta}, H_{z}$ on the lower plate is assumed to be zero here. In present investigation, induced magnetic field $\widehat{B}(r, \theta, z)$ having the component $\widehat{B}_{r}, \widehat{B}_{\theta}, \widehat{B}_{z}$ is generated by the magnetic field (applied) in a squeezed film between the plates. Figure 1 shows the geometrical coordinates. The upper and lower plate are held at fixed temperature $\left(T_{0}, T_{1}\right)$ and concentration $\left(C_{0}, C_{1}\right)$. The fluid is electrically conducting under the suspension of nanoparticles and gyrotactic microorganisms.

2.1. Rheological Model for Carreau Fluid. The Carreau fluid model is defined as [43]

$$
\tilde{\tau}_{i j}=\left[\mu_{\infty}+\left(\mu_{0}-\mu_{\infty}\right)\left(1+(\Gamma \dot{\gamma})^{2}\right)^{(n-1 / 2)}\right] \widehat{A}_{1},
$$

in which $\tilde{\tau}_{i j}$ represents the stress tensor, $\mu_{0}$ represents the zero shear rate viscosity, $\mu_{\infty}$ represents the limiting constant viscosity at infinite shear rate viscosity, $\Gamma$ represents the 


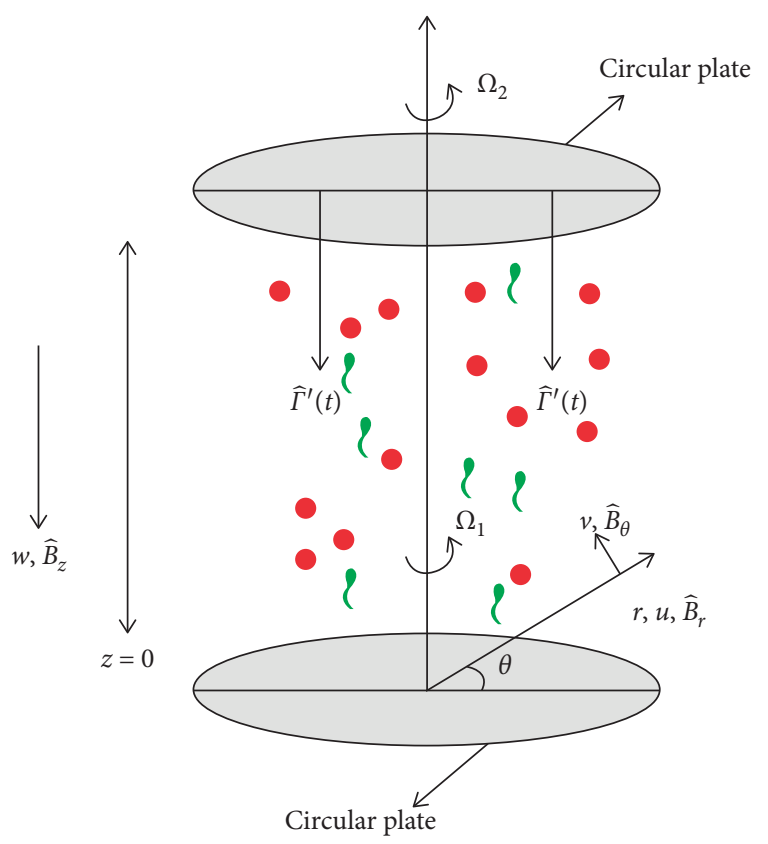

Nanoparticles

Microorganisms

FIGURE 1: The schematic diagram of nanoparticles between parallel finite plates in the presence of microorganisms.

constant of time, $n$ represents the power law index, $\widehat{A}_{1}$ represents the first Rivlin-Ericksen tensor, and $\dot{\gamma}$ represents the second invariant rate of strain tensor which is defined as

$$
\begin{aligned}
\dot{\gamma} & =\sqrt{\frac{1}{2} \operatorname{tr}\left(\widehat{A}_{1}\right)}, \\
\widehat{A}_{1} & =\left(L+L^{T}\right)=\left[\left(\nabla V+\nabla V^{T}\right)\right] .
\end{aligned}
$$

Assume that $\mu_{\infty}$ is zero, and by using binomial series approximation of first order of (2), we have

$$
\tilde{\tau}_{i j}=\left[\mu_{0}\left(1+\frac{n-1}{2}(\Gamma \dot{\gamma})^{2}\right)\right] \widehat{A}_{1} .
$$

The Carreau fluid model in component form is given in Appendix.

2.2. Problem Formulation. The governing flow equations by taking the above assumptions, the MHD squeezed film regime for Carreau fluid model, the equation of continuity, and momentum in $r, \theta, z$ direction can be read as

$$
\begin{aligned}
& \frac{1}{r} \frac{\partial}{\partial r}\left(r v_{r}\right)+\frac{1}{r} \frac{\partial}{\partial \theta}\left(v_{\theta}\right)+\frac{\partial}{\partial z}\left(v_{z}\right)=0 \\
& \rho\left(\frac{\partial v_{r}}{\partial t}+v_{r} \frac{\partial v_{r}}{\partial r}+\frac{v_{\theta}}{r} \frac{\partial v_{r}}{\partial \theta}+v_{z} \frac{\partial v_{r}}{\partial z}-\frac{v_{\theta}}{r}\right)=-\frac{\partial p}{\partial r} \\
& \quad+\frac{1}{r} \frac{\partial}{\partial r}\left(r \widetilde{\tau}_{r r}\right)+\frac{1}{r} \frac{\partial \widetilde{\tau}_{r \theta}}{\partial \theta}-\frac{\widetilde{\tau}_{\theta \theta}}{r}+\frac{\partial \widetilde{\tau}_{r z}}{\partial z}-\frac{\partial B_{r}}{\partial z} B_{z}-\frac{\partial B_{\theta}}{\partial z} B_{\theta} \\
& \rho\left(\frac{\partial v_{\theta}}{\partial t}+v_{r} \frac{\partial v_{\theta}}{\partial r}+\frac{v_{\theta}}{r} \frac{\partial v_{\theta}}{\partial \theta}+v_{z} \frac{\partial v_{\theta}}{\partial z}-\frac{v_{r} v_{\theta}}{r}\right)=-\frac{1}{r} \frac{\partial p}{\partial \theta} \\
& \quad+\frac{1}{r} \frac{\partial \widetilde{\tau}_{\theta \theta}}{\partial \theta}+\frac{1}{r^{2}} \frac{\partial\left(r^{2} \widetilde{\tau}_{r \theta}\right)}{\partial r}+\frac{\partial \widetilde{\tau}_{\theta z}}{\partial z}-\frac{\partial B_{\theta}}{\partial z} B_{z}-\frac{\partial B_{\theta}}{\partial r} B_{r} \\
& \rho\left(\frac{\partial v_{z}}{\partial t}+v_{r} \frac{\partial v_{z}}{\partial r}+\frac{v_{\theta}}{r} \frac{\partial v_{z}}{\partial \theta}+v_{z} \frac{\partial v_{z}}{\partial z}\right)=-\frac{\partial p}{\partial z} \\
& \quad+\frac{1}{r} \frac{\partial}{\partial r}\left(r \tilde{\tau}_{r z}\right)+\frac{1}{r} \frac{\partial \widetilde{\tau}_{\theta z}}{\partial \theta}+\frac{\partial \tilde{\tau}_{z z}}{\partial z}-\frac{\partial B_{\theta}}{\partial z} B_{\theta}+\frac{\partial B_{r}}{\partial z} B_{r}
\end{aligned}
$$

where $\rho, \mu, \widetilde{\tau}, p$ are denoted by fluid density, viscosity, stress tensor, and pressure, respectively. The equation of magnetic field can be read as

$$
\begin{gathered}
\frac{1}{r} \frac{\partial}{\partial r}\left(r B_{r}\right)+\frac{1}{r} \frac{\partial B_{\theta}}{\partial \theta}+\frac{\partial B_{z}}{\partial z}=0 \\
\frac{\partial B_{r}}{\partial t}+u \frac{\partial B_{r}}{\partial r}+v \frac{\partial B_{r}}{\partial \theta}+w \frac{\partial B_{r}}{\partial z}=-\frac{\partial}{\partial z}\left(v_{r} B_{z}-v_{z} B_{r}\right)+\frac{1}{\delta \mu_{2}}\left(\frac{\partial^{2} B_{r}}{\partial z^{2}}\right), \\
\frac{\partial B_{\theta}}{\partial t}+u \frac{\partial B_{\theta}}{\partial r}+v \frac{\partial B_{\theta}}{\partial \theta}+w \frac{\partial B_{\theta}}{\partial z}=\frac{\partial}{\partial r}\left(v_{r} B_{\theta}-v_{\theta} B_{r}\right)-\frac{\partial}{\partial z}\left(v_{\theta} B_{z}-B_{\theta} v_{z}\right)+\frac{1}{\delta \mu_{2}}\left(\frac{\partial^{2} B_{\theta}}{\partial z^{2}}\right), \\
\frac{\partial B_{z}}{\partial t}+u \frac{\partial B_{z}}{\partial r}+v \frac{\partial B_{z}}{\partial \theta}+w \frac{\partial B_{z}}{\partial z}=\frac{\partial}{\partial r}\left(v_{r} B_{z}-v_{z} B_{r}\right)+\frac{1}{\delta \mu_{2}}\left(\frac{\partial^{2} B_{z}}{\partial z^{2}}\right),
\end{gathered}
$$

where $\delta$ represents the electrical conductivity. 


$$
\frac{\partial \breve{T}}{\partial t}+v_{r} \frac{\partial \breve{T}}{\partial r}+v_{z} \frac{\partial \breve{T}}{\partial z}=\widetilde{\alpha} \frac{\partial^{2} \breve{T}}{\partial z^{2}}+\tau\left[D_{B}\left(\frac{\partial \breve{T}}{\partial r} \frac{\partial \breve{C}}{\partial r}+\frac{\partial \breve{T}}{\partial z} \frac{\partial \breve{C}}{\partial z}\right)+\frac{D_{T}}{T_{\infty}}\left[\left(\frac{\partial \breve{T}}{\partial r}\right)^{2}+\left(\frac{\partial \breve{T}}{\partial z}\right)^{2}\right]\right]
$$

where $\breve{T}, \breve{C}, \breve{T}_{m}, D_{B}, \tau=(\rho c)_{f} /(\rho c)_{p}, \widetilde{\alpha}=k /(\rho c)_{p} D_{T}$ are the temperature, concentration, mean fluid temperature, Brownian diffusivity, the proportion of the effected heat capacitance of the nanoparticle to the base fluid, and thermophoretic diffusion coefficient, respectively, where $k$ is the thermal conductivity and $(\rho c)_{p}$ is the heat capacity of the nanofluid.

The concentration of nanoparticle equation with activation energy reads

$$
\begin{aligned}
\frac{\partial \breve{C}}{\partial t}+v_{r} \frac{\partial \breve{C}}{\partial r}+v_{z} \frac{\partial \breve{C}}{\partial z}= & D_{B} \frac{\partial^{2} \breve{C}}{\partial z^{2}}+\frac{D_{T}}{\breve{T}} \frac{\partial^{2} \breve{T}}{\partial z^{2}}-k_{r}^{2}\left(C-C_{\infty}\right)\left(\frac{\breve{T}}{\breve{T}_{\infty}}\right)^{\bar{n}} \\
& \cdot \exp \left(-\frac{E_{a}}{1+\kappa \bar{T}}\right),
\end{aligned}
$$

where $\kappa, k_{r}^{2}, \bar{n}$, and $E_{a}$ represent the Boltzmann constant, reaction rate, rate constant, and activation energy, respectively.

The conservation of microorganism reads

$$
\begin{aligned}
\frac{\partial n}{\partial t} & +v_{r} \frac{\partial n}{\partial r}+v_{\theta} \frac{\partial n}{\partial \theta}+v_{z} \frac{\partial n}{\partial z}+\frac{\bar{b} W_{m o}}{C_{\infty}}\left[\frac{\partial}{\partial z}\left(n \frac{\partial \breve{C}}{\partial z}\right)\right] \\
& =D_{m o}\left(\frac{\partial^{2} n}{\partial z^{2}}\right),
\end{aligned}
$$

where chemotaxis constant is combined with maximal speed of cell swimming and is denoted by $\bar{b} W_{\text {mo }}\left(\bar{b} W_{\text {mo }}\right.$ is considered as a constant) and diffusivity of microorganisms is denoted by $D_{\text {mo }}$.

The initial and boundary conditions for (5)-(15) with our assumptions are the same as considered in [44]

$$
\begin{gathered}
u=0, v=\Omega_{1} r \frac{D^{2}}{\widehat{\Gamma}^{2}(t)}, w=0, B_{z}=B_{\theta}=0, C=\breve{C}_{l}, T=\breve{T}_{l}, n=n_{l} \text { at } z=0, \\
\left\{\begin{array}{l}
u=0, v=\Omega_{2} r \frac{D^{2}}{\widehat{\Gamma}^{2}(t)}, B_{\theta}=N_{0} r \frac{D^{2}}{\widehat{\Gamma}^{2}(t)}, B_{z}=-\frac{\beta D M_{0}}{\widehat{\Gamma}(t)}, w=-\frac{\beta D^{2}}{2 \widehat{\Gamma}(t)}, \quad \text { at } z=\widehat{\Gamma}(t), \\
T=\breve{T}_{u}, C=\breve{C}_{u}, n=n_{u},
\end{array}\right.
\end{gathered}
$$

2.3. Similarity Transformations. The set of similarity transformations are introduced as

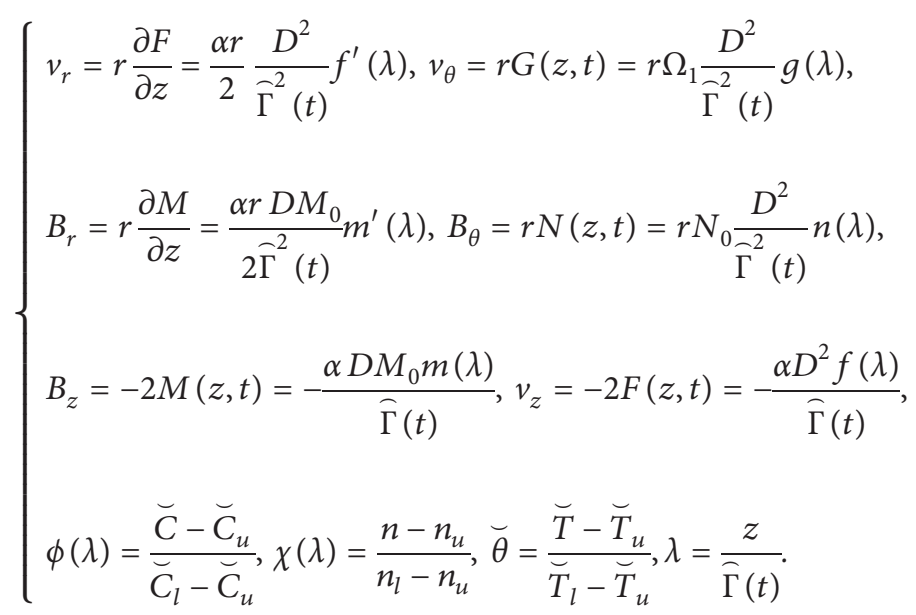

Now substituting the abovementioned similarity transformation in (5)-(15), the following highly coupled nonlinear ordinary differential equations (ODEs) with unitspaced variable $\lambda$ are obtained as 


$$
\begin{aligned}
& f^{(i v)}=8 S_{Q}\left[3 f^{\prime \prime}-2\left(\frac{R_{\Omega}}{S_{Q}}\right)^{2} g g^{\prime}+2 F_{T}^{2}\left(m m^{\prime \prime \prime}+m^{\prime} m^{\prime \prime}\right)-(2 f-\lambda) f^{\prime \prime \prime}+2 F_{A}^{2}\left(\frac{R_{\Omega}}{S_{Q}}\right)^{2} n n^{\prime}\right] \\
& -4(n-1) W e\left[\begin{array}{c}
\frac{1}{S_{Q}}\left(2 f^{\prime} g^{\prime} g^{\prime \prime}+f^{\prime \prime} g^{\prime 2}\right)+\frac{S_{Q}}{R_{\Omega}^{2}}\left[7 f^{\prime} f^{\prime \prime} f^{\prime \prime \prime}+3 f^{\prime \prime 3}+\frac{1}{2} f^{\prime 2} f^{i v}-\frac{3 R e}{4 R_{\Omega}}\left(2 f^{\prime \prime} f^{\prime \prime \prime 2}+f^{\prime \prime 2} f^{i v}\right)\right] \\
-\frac{\operatorname{Re}}{2 R_{\Omega} S_{Q}}\left[\left(g^{\prime} f^{\prime \prime} g^{\prime \prime \prime}+g^{\prime} f^{\prime \prime \prime} g^{\prime \prime}+f^{\prime \prime} g^{\prime \prime 2}\right)-\frac{1}{4}\left(g^{\prime 2} f^{i v}+2 g^{\prime} g^{\prime \prime} f^{\prime \prime \prime}\right)\right]
\end{array}\right], \\
& g^{\prime \prime}(\eta)=2 S_{Q}^{2}\left[2 g+\lambda g^{\prime}+2 g f^{\prime}-f g^{\prime}+2 F_{A} F_{T}\left(m n^{\prime}+n m^{\prime}\right)\right] \\
& -(n-1) W e S_{Q}\left[\frac{2 S_{Q}}{R_{\Omega}^{2}}\left(f^{\prime 2} g^{\prime \prime}+2 f^{\prime} f^{\prime \prime} g^{\prime}\right)-\frac{3 R e}{2 S_{Q} R_{\Omega}} g^{\prime 2} g^{\prime \prime}-\frac{\operatorname{Re}_{Q}}{R_{Q}^{3}}\left(\frac{3 f^{\prime \prime 2} g^{\prime \prime}}{4}+g^{\prime} f^{\prime \prime} f^{\prime \prime \prime}\right)\right] \text {, } \\
& m^{\prime \prime}=\operatorname{Re}_{M}\left[m+\lambda m^{\prime}+2 m f^{\prime}-2 f m^{\prime}\right] \\
& n^{\prime \prime}=\operatorname{Re}_{M}\left[2 n-f n^{\prime}+\lambda n^{\prime}+2\left(\frac{F_{A}}{F_{T}}\right) m g^{\prime}\right], \\
& \breve{\theta}^{\prime \prime}+S_{Q} P_{t} f \overline{\theta^{\prime}}+T_{t} \theta^{\prime \prime 2}+T_{b} \smile \theta^{\prime} \phi^{\prime}=0, \\
& \phi^{\prime \prime}+\frac{T_{t}}{T_{b}} \breve{\theta}^{\prime \prime}+S_{Q} S_{M} f \phi^{\prime}-S_{M} \sigma(1+\widetilde{\delta} \breve{\theta})^{\bar{n}} \exp \left(-\frac{E}{1+\widetilde{\delta} \theta}\right) \phi=0 \\
& \chi^{\prime \prime}-S_{\mathrm{Q}} B_{s}\left(\frac{\lambda}{2}\right) \chi^{\prime}+B_{s} S_{\mathrm{Q}} f \chi^{\prime}-P_{l}\left[\chi^{\prime} \phi^{\prime}+(\chi+\Phi) \phi^{\prime \prime}\right]=0
\end{aligned}
$$

where squeezed Reynolds number is represented by $S_{Q}$, rotational Reynolds number is denoted by $R_{\Omega}$, magnetic field strength in axial and azimuthal direction is represented by $F_{A}, F_{T}$, respectively, magnetic Reynolds number is represented by $\operatorname{Re}_{M}$, Weissenberg number is represented by $W e$, Brownian motion parameter is represented by $T_{b}$, thermophoresis parameter is represented by $T_{t}$, Prandtl number is represented by $P_{t}$, Schmidt number is represented by $S_{M}$, chemical reaction parameter is represented by $\sigma$, temperature difference is represented by $\widetilde{\delta}$, bioconvection Schmidt number is represented by $B_{s}$, Peclet number is represented by $P_{l}$, and $\Phi$ represents constant number. They are defined as

$$
\left\{\begin{array}{l}
S_{Q}=\frac{\alpha D^{2}}{2 v}, R_{\Omega}=\frac{\Omega_{1} D^{2}}{v}, F_{T}=\frac{M_{0}}{D \sqrt{\mu_{2} \rho}}, F_{A}=\frac{N_{0}}{\Omega_{1} \sqrt{\mu_{2} \rho}}, W e=\Omega_{1}^{2} \Gamma^{2} \\
T_{b}=\frac{\tau D_{B}\left(\breve{C}_{l}-\breve{C}_{u}\right)}{\widetilde{\alpha}}, T_{t}=\frac{\tau D_{T}\left(\breve{T}_{l}-\breve{T}_{u}\right)}{\widetilde{\alpha}}, P_{t}=\frac{v}{\widetilde{\alpha}}, S_{M}=\frac{v}{D_{B}} \\
B_{s}=\frac{v}{D_{n}}, P_{l}=\frac{\bar{b} W_{m o}}{D_{m o}}, \Phi=\frac{n_{\infty}}{n_{w}-n_{\infty}}, B t=\delta \mu_{2} v, \operatorname{Re}_{M}=R_{Q} B t
\end{array}\right.
$$

where $B t$ is the Batchelor number.

The corresponding boundary mentioned in (16) and (17) is reduced as 


$$
\left\{\begin{array}{l}
f(0)=0=f^{\prime}(0), g(0)=1, m(0)=0, n(0)=1, \breve{\theta}(0)=1, \chi(0)=1, \phi(0)=1, \\
f(1)=\frac{1}{2}, g(1)=\dot{\xi}, m(1)=1, n(1)=1, \breve{\theta}(1)=0, \phi(1)=0, \chi(1)=0,
\end{array}\right.
$$

where $f$ represents the axial and $g$ represents the tangential velocity, $m$ represents the axial and $n$ represents the tangential induced magnetic field components, $\theta$ represents the temperature profile, $\phi$ represents nanoparticles concentration, $\chi$ represents the motile density microorganism profile, and $\xi\left(=\Omega_{2} / \Omega_{1}\right)$ denotes the angular velocity and the range of the velocity betwixt the rotating plates is $-1 \leq \dot{\xi} \leq 1$, which is useful to analyze various flows characteristics of rotating plates which revolve in the same or opposite directions.

The dimensionless torque can be measured on the moving disk by

$$
\dot{T}_{\text {up }}=2 \pi \rho \int_{0}^{b}\left(\frac{\partial v}{\partial z}\right)_{z=\bar{\Gamma}(t)} \mathrm{d} r .
$$

The radius of the disk is denoted by $b$.

Using (18) in (28), it becomes

$$
\dot{T}_{\text {up }}=\frac{\mathrm{d} g(1)}{\mathrm{d} \lambda}
$$

where the nondimensional torque on moving disk by fluid is denoted by $\dot{T}_{\text {up }}$, and gradient of the tangential velocity on the moving disk is $\mathrm{d} g(1) / \mathrm{d} \lambda$.

Similarly, the torque in dimensionless form on the lower (fixed) plate is obtained by the same calculation at $\lambda=0$; it becomes

$$
\dot{T}_{l p}=\frac{\mathrm{d} g(0)}{\mathrm{d} \lambda}
$$

\section{Solution of the Problem: Differential Transform Method (DTM)}

The nonlinear dimensionless ordinary differential equations (19)-(25) with boundary conditions (28) are elucidated with DTM. The DTM produces an analytical result based on Taylor series expansion in polynomial form. The differential transform method can easily be applied to linear or nonlinear problems and reduces the size of computational work. With this method, exact solutions may be obtained without cumbersome work, and it is a useful tool for analytical and numerical solutions. In the past decades, DTM has been successfully applied in many models of fluid dynamics, nanofluid dynamics in biotechnology, heat transfer, burgers equations, applications to nonlinear oscillators, plane Couette fluid flow problem, free vibration analysis, micropolar fluid flow, and non-Newtonian nanofluids flow analysis [45-48]. The proposed Padé approximation helps to enhance the convergence rate of the solutions of the truncated series. The DTM solutions can not satisfy the given boundary conditions at infinity without the use of Padé approximations. It is, therefore, essential to use DTM-Padé to afford an effective way to deal with infinite boundary value problems. Mathematica (12v) software has been used to obtain approximations. The complete procedure of this method is described by Zhang et al. [49]. Taking differential transform of each term of (19)-(25), the following transformations are obtained:

$$
\left.\begin{array}{rl}
f^{\prime \prime} \longrightarrow & (k+1)(2+k) f(2+k), \\
f^{\prime \prime 3} \longrightarrow & {\left[\sum_{m=0}^{k}\left(\begin{array}{c}
\sum_{r=0}^{m}(r+1)(r+2)(-r+m+1)(-m+k+1)(-m+k+2) \\
f(-m+k+2) f(2+r) f(-r+2+k)
\end{array}\right)\right],} \\
f^{\prime} f^{\prime \prime} f^{\prime \prime \prime} & \longrightarrow\left[\sum_{r=0}^{k}\left(\begin{array}{c}
\sum_{m=0}^{k-r}(r+1)(1+m)(2+m)(-r+k-m+1)(-r+2+k-m) \\
(-m+k-r+3) f(1+r) f(2+m) f(-r+k-m+3)
\end{array}\right)\right], \\
f^{\prime \prime} f^{\prime \prime \prime 2} & \longrightarrow\left[\sum_{r=0}^{k}\left(\begin{array}{c}
k-r \\
(-m+k+2-r)(-m+k-r+3) f(3+r) f(2+m) f(-m+k-r+3)
\end{array}\right)\right] \\
g & (k), \\
\lambda g^{\prime} \longrightarrow \sum_{r=0}^{k}((-r+1+k) \varepsilon(r) g(-r+1+k)),
\end{array}\right\},
$$




$$
\begin{aligned}
& g f^{\prime} \longrightarrow \sum_{r=0}^{k}(-r+1+k) g(r) f(-r+1+k) \\
& f g^{\prime} \longrightarrow \sum_{r=0}^{k}(-r=1+k) f(r) g(-r+1+k) \\
& f^{\prime} g^{\prime} g^{\prime \prime} \longrightarrow \sum_{r=0}^{k}(r+1)(-r+1+k)(-r+2+k) f(1+r) g(1+r) g(-r+2+k), \\
& g^{\prime} g^{\prime \prime} f^{\prime \prime \prime} \longrightarrow \sum_{r=0}^{k}(1+r)(r+2)(-r+1+k)(-r+2+k)(-r+k+3) g(1+r) g(2+r) \\
& g(-r+k+3) \text {, } \\
& f^{\prime} g^{\prime} f^{\prime \prime} \longrightarrow \sum_{r=0}^{k}(1+r)(-r+k+1)(-r+2+k) f(1+r) g(1+r) f(-r+2+k) \text {, } \\
& g^{\prime} f^{\prime \prime} f^{\prime \prime \prime} \longrightarrow \sum_{r=0}^{k}(1+r)(2+r)(-r+1+k)(-r+2+k)(-r+k+3) g(r+1) f(2+r) \\
& f(-r+3+k) \text {, } \\
& f^{\prime \prime} g^{\prime 2} \longrightarrow \sum_{m=0}^{k}\left(\begin{array}{c}
\sum_{r=0}^{m}(1+r)(2+r)(1-r+m)(-m+1+k) g(-m+1+k) f(2+r) \\
g(-r+1+k)
\end{array}\right), \\
& f^{\prime \prime} g^{\prime \prime 2} \longrightarrow \sum_{r=0}^{k}\left(\begin{array}{c}
\sum_{m=0}^{k-r}(r+1)(2+r)(m+1)(m+2)(-m+1-r+k)(-r+2+k-m) \\
g(2+r) f(m+2) g(-r+2+k-m)
\end{array}\right) \\
& {f^{\prime}}^{2} g^{\prime \prime} \longrightarrow \sum_{m=0}^{k}\left(\begin{array}{c}
\sum_{r=0}^{m}(1+r)(2+r)(-r+1+m)(-m+1+k) f(-m+1+k) g(2+r) \\
f(-r+k+1)
\end{array}\right) \\
& m^{\prime} m^{\prime \prime} \longrightarrow \sum_{r=0}^{k}(r+1)(2+r)(-r+1+k)(-r+2+k) m(r+1) m(-r+2+k), \\
& \lambda m^{\prime} \longrightarrow \sum_{r=0}^{k}((-r+1+k) \mathcal{\varepsilon}(r) m(-r+k+1)) \\
& m f^{\prime} \longrightarrow \sum_{r=0}^{k}((-r+1+k) m(r) f(-r+1+k)) \text {, } \\
& f m^{\prime} \longrightarrow \sum_{r=0}^{k}((-r+1+k) f(r) m(-r+1+k)) \text {, } \\
& m g^{\prime} \longrightarrow \sum_{r=0}^{k}((-r+1+k) m(r) g(-r+1+k)) \text {, } \\
& n n^{\prime} \longrightarrow \sum_{r=0}^{k}((-r+1+k) n(r) n(-r+1+k)) \\
& f n^{\prime} \longrightarrow \sum_{r=0}^{k}((-r+1+k) f(r) n(-r+1+k)) \text {, } \\
& \lambda n^{\prime} \longrightarrow \sum_{r=0}^{k}((-r+1+k) \varepsilon(r) n(-r+1+k)),
\end{aligned}
$$




$$
\left.\begin{array}{c}
f \backsim \theta^{\prime} \longrightarrow \sum_{r=0}^{k}((-r+1+k) f(r) \breve{\theta}(-r+1+k)), \\
\breve{\theta}^{\prime 2} \longrightarrow \sum_{r=0}^{k}((1+r)(-r+1+k) \breve{\theta}(1+r) \breve{\theta}(1-r+k)),
\end{array}\right\}
$$

where the transformed functions of $f(\lambda), g(\lambda), m(\lambda)$, $n(\lambda), \theta(\lambda), \phi(\lambda)$ and $\chi(\lambda)$ are $f(k), g(k), m(k), n(k), \theta(k)$, $\phi(k)$ and $\chi(k)$, respectively, and are expressed as

$$
\begin{aligned}
& f(\lambda)=\sum_{k=0}^{\infty} f(k) \lambda^{k}, \\
& g(\lambda)=\sum_{k=0}^{\infty} g(k) \lambda^{k}, \\
& m(\lambda)=\sum_{k=0}^{\infty} m(k) \lambda^{k}, \\
& n(\lambda)=\sum_{k=0}^{\infty} n(k) \lambda^{k}, \\
& \breve{\theta}(\lambda)=\sum_{k=0}^{\infty} \breve{\theta}(k) \lambda^{k}, \\
& \phi(\lambda)=\sum_{k=0}^{\infty} \phi(k) \lambda^{k}, \\
& \chi(\lambda)=\sum_{k=0}^{\infty} \chi(k) \lambda^{k} .
\end{aligned}
$$

The boundary conditions are

$$
\begin{aligned}
& f(0)=0, f(1)=\frac{1}{2}, g(0)=1, m(0)=0, n(0)=0, \\
& \breve{\theta}(0)=1, \phi(0)=0, \chi(0)=0, f(2)=\Pi_{1}, f(3)=\Pi_{2}, \\
& g(1)=\Pi_{3}, m(1)=\Pi_{4}, n(1)=\Pi_{5}, \breve{\theta}(1)=\Pi_{6}, \phi(1)=\Pi_{6}, \\
& \chi(1)=\Pi_{8} .
\end{aligned}
$$

Substituting transformations given in (31)-(38) into (19)-(25) and solving with the help of boundary conditions given in (40), the series solutions are

$$
\begin{aligned}
& f(\lambda)=\bar{f}_{1} \lambda^{2}+\bar{f}_{2} \lambda^{3}+\bar{f}_{3} \lambda^{4}+\bar{f}_{4} \lambda^{5}+\ldots, \\
& g(\lambda)=1-\bar{g}_{1} \lambda+\bar{g}_{2} \lambda^{2}+\bar{g}_{3} \lambda^{3}+\bar{g}_{4} \lambda^{4}+\ldots, \\
& m(\lambda)=\bar{m}_{1} \lambda+\bar{m}_{2} \lambda^{3}+\bar{m}_{3} \lambda^{4}+\bar{m}_{4} \lambda^{5}+\ldots, \\
& n(\lambda)=\bar{n}_{1} \lambda+\bar{n}_{2} \lambda^{3}+\bar{n}_{3} \lambda^{4}+\bar{n}_{4} \lambda^{5}+\ldots, \\
& \bar{\theta}(\lambda)=1+\bar{\theta}_{1} \lambda+\bar{\theta}_{2} \lambda^{2}+\bar{\theta}_{3} \lambda^{3}+\bar{\theta}_{4} \lambda^{4}+\ldots, \\
& \phi(\lambda)=1+\bar{\phi}_{1} \lambda+\bar{\phi}_{2} \lambda^{2}+\bar{\phi}_{3} \lambda^{3}+\bar{\phi}_{4} \lambda^{4}+\ldots, \\
& \chi(\lambda)=1+\bar{\chi}_{1} \lambda+\bar{\chi}_{2} \lambda^{2}+\bar{\chi}_{3} \lambda^{3}+\bar{\chi}_{4} \lambda^{4}+\ldots,
\end{aligned}
$$

where $\bar{f}_{i}, \bar{g}_{i}, \bar{m}_{i}, \bar{n}_{i}, \bar{\theta}_{i}, \bar{\phi}_{i} \bar{\chi}_{i} ; \quad(i=1,2,3, \ldots)$ are constants but difficult to represent here. With the aid of Mathematica $(12 \mathrm{v})$ software, the above equations are solved with 30 iterations. However, the rate of convergence is not obtained. Some schemes are available to increase the rate of convergence. One of the easiest ways to enhance the rate of convergence of the truncated series is Padé approximation, which is utilized into the form of rational fraction (ratio of two polynomials). Without the use of Padé approximation, the results attained by DTM do not satisfy the boundary condition at infinity because of the nonlinearity in the governing equations. So it is compulsory to combine the analytic solution obtained by DTM with the Padé approximation which gives good convergence rate at infinity. As a result of numerical values to a desired exactness, the number of terms required is determined by the higher approximation 
of the order. The Padé approximation of order [5×5] is applied to (41)-(47); the Padé approximants are as follows:

$$
\begin{aligned}
f(\lambda) & =\frac{1.90345 \lambda^{2}-1.46791 \lambda^{3}+0.305748 \lambda^{4}-0.096132 \lambda^{5}+\ldots}{1+0.254218 \lambda+0.054450 \lambda^{2}-0.00576 \lambda^{3}-0.0106312 \lambda^{4}-0.00369678 \lambda^{5}+\ldots}, \\
g(\lambda) & =\frac{1-2.5321 \lambda+0.1463087 \lambda^{2}+0.8214957 \lambda^{3}+0.4412932 \lambda^{4}+0.130549 \lambda^{5}}{1-1.53185 \lambda-1.38353 \lambda^{2}-0.56653 \lambda^{3}-0.1264778 \lambda^{4}-0.0004279 \lambda^{5}}, \\
m(\lambda) & =\frac{0.70958 \lambda+0.088916 \lambda^{2}-0.013768 \lambda^{3}+0.301618 \lambda^{4}-0.3283745 \lambda^{5}}{1+0.12530 \lambda-0.352736 \lambda^{2}+0.066053 \lambda^{3}-0.0612512 \lambda^{4}-0.019786 \lambda^{5}} \\
(\lambda) & =\frac{0.93923 \lambda+1.48352 \lambda^{2}+0.904482 \lambda^{3}+0.462497 \lambda^{4}+0.191811 \lambda^{5}}{1+1.579508 x+0.7148985 x^{2}+0.418387 \lambda^{3}+0.296779 x^{4}-0.022257 \lambda^{5}} \\
\varphi(\lambda) & =\frac{1-0.80937 \lambda-0.215262 \lambda^{2}+0.013511 \lambda^{3}+0.004125 \lambda^{4}+0.00701 \lambda^{5}}{1+0.12854 \lambda-0.0271242 \lambda^{2}-0.003919 \lambda^{3}-0.010742 \lambda^{4}+0.0005623 \lambda^{5}}, \\
\phi(\lambda) & =\frac{1-0.121238 \lambda-0.3440942 \lambda^{2}-0.48574198 \lambda^{3}-0.000109 \lambda^{4}-0.04746 \lambda^{5}}{1+1.059822 \lambda+0.6555562 \lambda^{2}+0.09425 \lambda^{3}+0.022888 \lambda^{4}+0.001157 \lambda^{5}} \\
\chi(\lambda) & =\frac{1-0.1473267 \lambda-0.6259649 \lambda^{2}-0.1604094 \lambda^{3}-0.0551968 \lambda^{4}-0.011072 \lambda^{5}}{1+0.9192844 \lambda+0.266335 \lambda^{2}+0.072151 \lambda^{3}+0.015844 \lambda^{4}+0.00046357 \lambda^{5}} .
\end{aligned}
$$

\section{Discussion of Numerical and Graphical Results}

The numerical results of DTM-Padé, which is employed to solve nonlinear coupled ordinary differential equations for the governing flow, are discussed in this section. Computational software Mathematica $(12 v)$ is used to obtain the solutions of the system of equations. We compare our results with the shooting method [50] to verify the accuracy of the present method. Our primary purpose is to analyze the physical significance of various parameters involved in the energy equation, induced MHD equation, nanoparticle concentration equation, momentum equation, and motile microorganism function. Multiple parameters are discussed, i.e., squeezing Reynolds number $S_{Q}$, rotational Reynolds number $R_{\Omega}$, Weissenberg number $W e$, Reynolds number Re, magnetic Reynolds number $\operatorname{Re}_{M}$ on velocity profiles $\left\{f^{\prime}(\lambda), g^{\prime}(\lambda)\right\}$, and induced magnetic field $\{m(\lambda), n(\lambda)\}$. Furthermore, the influence of squeezing Reynolds number $S_{Q}$, Prandtl number $P_{t}$, thermophoresis parameter $T_{t}$, Brownian motion $T_{b}$, activation energy $E$, activation rate $\sigma$, Schmidt number $S_{M}$, bioconvection number $B_{s}$, and Peclet number is also discussed. The numerical results for local Nusselt number, local Sherwood number, and motile density number are presented in Tables 1-3. From these tables, it is observed that our outcomes are in good agreement with the numerical shooting method. Equations (28)-(29) are used to calculate the effects of torque on the fixed and moving plate, which is displayed in Table 4. For the graphical outcomes, we have selected the following parametric values against each parameter: $S_{M}=0.2, P e=0.5, B_{s}=5, S_{Q}=0.02, R_{\Omega}=0.2$,
$W e=0.01, \mathrm{Re}=0.001, \operatorname{Re}_{M}=0.5, \quad T_{t}=0.03, T_{b}=0.1$, $P_{t}=6.8, E=2, \sigma=2$.

Figure 2 shows the impact on the velocity field in the axial direction $f^{\prime}(\lambda)$ due to squeezed Reynolds number $S_{Q}$ and rotational Reynolds number $R_{\Omega}$. It is observed when the values of squeezed Reynolds number $S_{Q}$ enhance, the axial velocity distribution decreases. However, when increasing the values of $R_{\Omega}$, the axial velocity $f^{\prime}(\lambda)$ increases. This is because the rotation of the plates accelerates the velocity of the fluid. Furthermore, in Figure 3, it is seen that when increasing value of $\mathrm{Re}$, the velocity of the fluid declines and the Weissenberg number $W e$ increases the axial velocity profile. Physically, when the values of the Weissenberg number $W e$ are increased, the relaxation time increases for the particles of Carreau fluid, so these particles and velocity decrease the resistance. This is because the Weissenberg number $W e$ is the ratio of relaxation time of the fluid and particular process time. In Figure 4, the impact of Weissenberg number on Carreau fluid velocity for shear-thinning $(n<1)$, Newtonian case $(n=0)$, and thickening $(n>1)$ cases can be seen on axial velocity distribution $f^{\prime}(\lambda)$. This is because, in the shear-thinning case, the fluid viscosity is lower with shear strain, and the velocity is high compared to the Newtonian and shear-thickening case. Figures 5 and 6 represent the influence of squeezed Reynolds number and rotational Reynolds number on tangential velocity distribution $g^{\prime}(\lambda)$. It is observed that, by enhancing the value of squeezed Reynolds number and rotational Reynolds number, the tangential velocity distribution decreases, but the effects are negligible. From Figure 7, it is noticed that the axial and tangential induced magnetic field distributions 
TABLE 1: Comparison of Nusselt number $-\theta^{\prime}(0)$ for various values $T_{t}, T_{b}, P_{t}, S_{Q}$ by shooting method and DTM-Padé [5 $\times 5$ ].

\begin{tabular}{|c|c|c|c|c|c|c|c|c|c|}
\hline \multirow[b]{2}{*}{$T_{t}$} & \multirow[b]{2}{*}{$T_{b}$} & \multirow[b]{2}{*}{$P_{t}$} & \multirow[b]{2}{*}{$S_{Q}$} & \multicolumn{2}{|c|}{$n=-3, W e=0.01$} & \multicolumn{2}{|c|}{$n=1, W e=0$} & \multicolumn{2}{|c|}{$n=3, W e=0.01$} \\
\hline & & & & $\begin{array}{l}\text { Shooting } \\
\text { method }\end{array}$ & DTM-Padé & Shooting method & DTM-Padé & $\begin{array}{l}\text { Shooting } \\
\text { method }\end{array}$ & DTM-Padé \\
\hline 0.03 & 0.01 & 6.8 & 0.05 & 0.89308203 & 0.89308203 & 0.89309699 & 0.89309699 & 0.89310474 & 0.89310474 \\
\hline 0.06 & & & & 0.80302479 & 0.80302479 & 0.80303833 & 0.80303833 & 0.80304535 & 0.80304535 \\
\hline 0.09 & & & & 0.71985201 & 0.71985201 & 0.71986417 & 0.71986417 & 0.71987048 & 0.71987048 \\
\hline 0.05 & 0.1 & & & 0.58574689 & 0.58574689 & 0.58575671 & 0.58575671 & 0.5857618 & 0.5857618 \\
\hline & 0.3 & & & 0.24295587 & 0.25072139 & 0.24295986 & 0.24295986 & 0.24296193 & 0.24296193 \\
\hline & 0.5 & & & 0.08998096 & 0.08998096 & 0.08998239 & 0.08998239 & 0.08998315 & 0.08998315 \\
\hline & 0.01 & 4 & & 0.89845222 & 0.89845222 & 0.89846105 & 0.89846105 & 0.89846562 & 0.89846562 \\
\hline & & 7 & & 0.82769241 & 0.82769241 & 0.82770676 & 0.82770676 & 0.82771419 & 0.82771419 \\
\hline & & 10 & & 0.76139836 & 0.76139836 & 0.76141735 & 0.76141735 & 0.76142719 & 0.76142719 \\
\hline & & 6.8 & -0.05 & 0.78688575 & 0.78688575 & 0.78688221 & 0.78688221 & 0.78688033 & 0.78688033 \\
\hline & & & 0.02 & 0.81862905 & 0.81862905 & 0.81863007 & 0.81863007 & 0.81863059 & 0.81863059 \\
\hline & & & 0.12 & 0.97452857 & 0.97452857 & 0.86421817 & 0.86421817 & 0.85056260 & 0.85056260 \\
\hline
\end{tabular}

TABLE 2: Comparison of $\chi^{\prime}(0)$ for various values of $S_{Q}, B_{s},{ }_{P}$ by shooting method and DTM-Padé [5 $\left.\times 5\right]$.

\begin{tabular}{|c|c|c|c|c|c|c|c|c|c|c|c|}
\hline \multirow[b]{2}{*}{$T_{t}$} & \multirow[b]{2}{*}{$T_{b}$} & \multirow[b]{2}{*}{$S_{\mathrm{Q}}$} & \multirow[b]{2}{*}{$S_{M}$} & \multirow[b]{2}{*}{$E$} & \multirow[b]{2}{*}{$\sigma$} & \multicolumn{2}{|c|}{$n=-3, W e=0.01$} & \multicolumn{2}{|c|}{$n=1, W e=0$} & \multicolumn{2}{|c|}{$n=3, W e=0.01$} \\
\hline & & & & & & Shooting method & DTM-Padé & Shooting method & DTM-Padé & Shooting method & DTM-Padé \\
\hline 0.03 & 0.01 & 0.05 & 5 & 3 & 2 & -1.48750051 & -1.48750051 & -1.48747228 & -1.48747228 & -1.48745764 & -1.48745764 \\
\hline 0.06 & & & & & & -2.31230089 & -2.31230089 & -2.31224459 & -2.31224459 & -2.31221539 & -2.31221539 \\
\hline 0.09 & & & & & & -3.59230155 & -3.59230155 & -3.59222994 & -3.59222994 & -3.59219276 & -3.59219276 \\
\hline 0.05 & 0.1 & & & & & -1.37683310 & -1.37683310 & -1.37684321 & -1.37684321 & -1.60791008 & -1.60791008 \\
\hline & 0.3 & & & & & -1.29862771 & -1.29862771 & -1.29864147 & -1.29864147 & -1.40562686 & -1.40562686 \\
\hline & 0.5 & & & & & -1.26476393 & -1.26476393 & -1.26477795 & -1.26477795 & -1.33606822 & -1.33606822 \\
\hline & 0.01 & -0.05 & & & & -2.16407621 & -2.16407621 & -2.16408297 & -2.16408297 & -3.91679239 & -3.91679239 \\
\hline & & 0.02 & & & & -2.03817454 & -2.03817454 & -2.03817048 & -2.03817048 & -3.69044429 & -3.69044429 \\
\hline & & 0.12 & & & & -1.96569720 & -1.96569720 & -1.85623506 & -1.85623506 & -3.49325834 & -3.49325834 \\
\hline & & 0.01 & 5 & & & -2.05625050 & -2.05625050 & -2.05624885 & -2.05624885 & -3.72303640 & -3.72303640 \\
\hline & & & 10 & & & -2.17949478 & -2.17949478 & -2.17949321 & -2.17949321 & -3.77225894 & -3.77225894 \\
\hline & & & 15 & & & -2.29665895 & -2.29665895 & -2.29665738 & -2.29665738 & -3.82221775 & -3.82221775 \\
\hline & & & 5 & 2 & & -1.99640130 & -1.99640130 & -2.81393318 & -2.81393318 & -3.69971651 & -3.69971651 \\
\hline & & & & 3 & & -1.95271335 & -1.95271335 & -2.29665738 & -2.29665738 & -3.68433831 & -3.68433831 \\
\hline & & & & 4 & & -1.93641884 & -1.93641884 & -2.07634697 & -2.07634697 & -3.67871779 & -3.67871779 \\
\hline & & & & 2 & 2 & -1.99640130 & -1.99640130 & -2.81393318 & -2.81393318 & -3.69971651 & -3.69971651 \\
\hline & & & & & 4 & -2.06392299 & -2.06392299 & -3.48018830 & -3.48018830 & -3.72438933 & -3.72438933 \\
\hline & & & & & 6 & -2.12957297 & -2.12957297 & -4.03630116 & -4.03630116 & -3.74942313 & -3.74942313 \\
\hline
\end{tabular}

TABLE 3: Comparison of $\phi^{\prime}(0)$ for various values $T_{t}, T_{b}, S_{Q}, E, \sigma$ by shooting method and DTM-Padé [5×5].

\begin{tabular}{|c|c|c|c|c|c|c|c|c|}
\hline \multirow[b]{2}{*}{$S_{Q}$} & \multirow[b]{2}{*}{$B_{s}$} & \multirow[b]{2}{*}{$P_{l}$} & \multicolumn{2}{|c|}{$n=-3, W e=0.01$} & \multicolumn{2}{|c|}{$n=1, W e=0$} & \multicolumn{2}{|c|}{$n=3, W e=0.01$} \\
\hline & & & Shooting method & DTM-Padé & Shooting method & DTM-Padé & Shooting method & DTM-Padé \\
\hline-0.05 & 5 & 0.5 & -3.68071264 & -3.68071264 & -3.68070328 & -3.68070328 & -3.68069877 & -3.68069877 \\
\hline 0.02 & & & -3.40398671 & -3.40398671 & -3.40397880 & -3.40397880 & -3.40397480 & -3.40397480 \\
\hline 0.12 & & & -3.24595020 & -3.24595020 & -3.20633134 & -3.20633134 & -3.20623463 & -3.20623463 \\
\hline 0.01 & 5 & & -3.44353509 & -3.44353509 & -3.44353113 & -3.44353113 & -3.44352914 & -3.44352914 \\
\hline & 10 & & -3.44262691 & -3.44262691 & -3.44262260 & -3.44262260 & -3.44262046 & -3.44262046 \\
\hline & 15 & & -3.44171898 & -3.44171898 & -3.44171434 & -3.44171434 & -3.44171203 & -3.44171203 \\
\hline & 1 & 0.5 & -3.23321118 & -3.23321118 & -3.23320693 & -3.23320693 & -3.23320481 & -3.23320481 \\
\hline & & 1.0 & -5.75926435 & -5.75926435 & -5.75925402 & -5.75925402 & -5.75924883 & -5.75924883 \\
\hline & & 1.5 & -8.53042052 & -8.53042052 & -8.53040286 & -8.53040286 & -8.53039401 & -8.53039401 \\
\hline
\end{tabular}

decline with increases in the value of magnetic Reynolds number $\operatorname{Re}_{M}$.

Figure 8 shows the influence of the thermophoresis parameter $T_{t}$ and the Brownian motion parameter $T_{b}$ on the temperature profile $\theta(\lambda)$ by keeping the rest of the parameters unaltered. The effect indicates that increasing the values of both parameters raises the temperature profile. The impact of $S_{Q}$ and Prandtl number $P_{t}$ is portrayed in Figure 9. It is seen that, by upgrading the Prandtl number $P_{t}$, the temperature profile $\breve{\theta}(\lambda)$ declines. The reason behind this is 
TABLE 4: Values of torque at the lower (fix) and upper (moving) plates.

\begin{tabular}{|c|c|c|c|c|c|c|c|}
\hline \multirow[b]{2}{*}{$S_{Q}$} & \multirow[b]{2}{*}{$R_{\Omega}$} & \multirow[b]{2}{*}{$W e$} & \multirow[b]{2}{*}{$\operatorname{Re}$} & \multicolumn{2}{|c|}{$\mathrm{d} g(0) / \mathrm{d} \lambda$} & \multicolumn{2}{|c|}{$\mathrm{d} g(1) / \mathrm{d} \lambda$} \\
\hline & & & & DTM-Padé & Shooting method & DTM-Padé & Shooting method \\
\hline 0.01 & 0.1 & 0.01 & 0.01 & -1.05591667 & -1.05591667 & -1.00586254 & -0.99994831 \\
\hline 0.03 & & & & -1.00101432 & -1.00101432 & -1.00688815 & -0.99954622 \\
\hline 0.05 & & & & -1.03595883 & -1.03595883 & -1.01803750 & -0.99874826 \\
\hline \multirow[t]{9}{*}{0.01} & 0.1 & & & -1.00101432 & -1.00101432 & -1.00586254 & -0.99994831 \\
\hline & 0.2 & & & -1.00087495 & -1.00087495 & -1.00030989 & -0.99989969 \\
\hline & 0.3 & & & -1.00091052 & -1.00091052 & -1.00062003 & -0.99988534 \\
\hline & 0.1 & 0.01 & & -1.00101432 & -1.00101432 & -1.00586254 & -0.99994831 \\
\hline & & 0.04 & & -1.00174207 & -1.00174207 & -1.02341830 & -1.00012927 \\
\hline & & 0.07 & & -1.00247679 & -1.00247679 & -1.04340432 & -1.00031195 \\
\hline & & 0.01 & 0.0013 & -1.00101290 & -1.00101290 & -1.00054533 & -0.99994839 \\
\hline & & & 0.0016 & -1.00101295 & -1.00101295 & -1.00053459 & -0.99994839 \\
\hline & & & 0.0019 & -1.00101299 & -1.00101299 & 1.00051846 & -0.99994839 \\
\hline
\end{tabular}

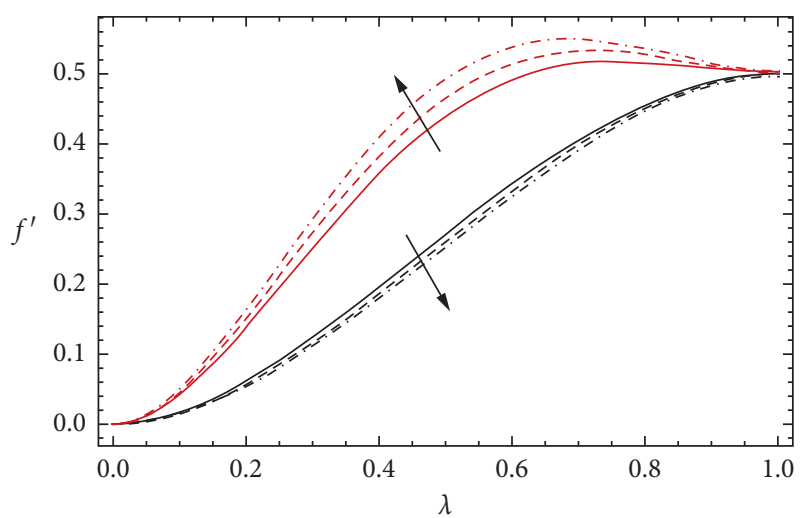

$$
\begin{aligned}
& -S_{Q}=-0.15,0.02,0.12 \\
& -R_{\Omega}=0.2,0.3,0.4
\end{aligned}
$$

Figure 2: Effect of various values of squeeze Reynolds number $S_{Q}$ and rotational Reynolds number $R_{\Omega}$ on axial velocity distribution $f^{\prime}(\lambda)$.

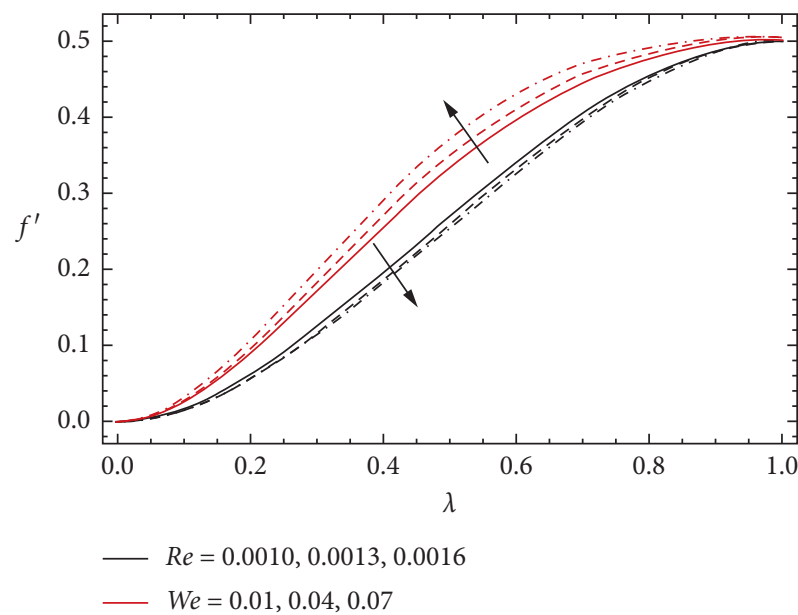

FIgURE 3: Effect of various values of Reynolds numberRe and Weissenberg number $W e$ on axial velocity distribution $f^{\prime}(\lambda)$.

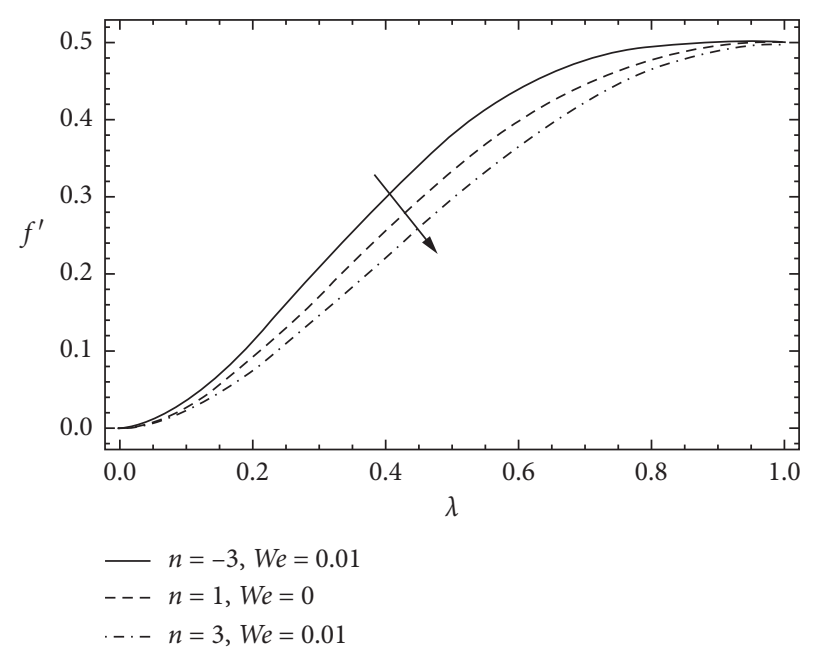

Figure 4: Effect of different values of power law index $n$ and Weissenberg number $W e$ on axial velocity distribution $f_{\prime}(\lambda)$.

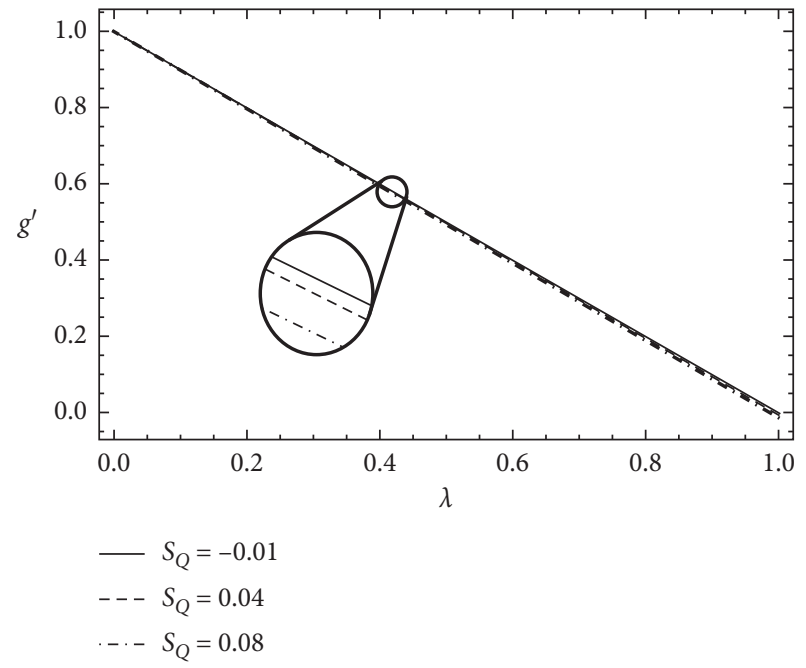

FIGURE 5: Effect of various values of squeeze Reynolds number $S_{Q}$ on the tangential velocity distribution $g^{\prime}(\lambda)$. 


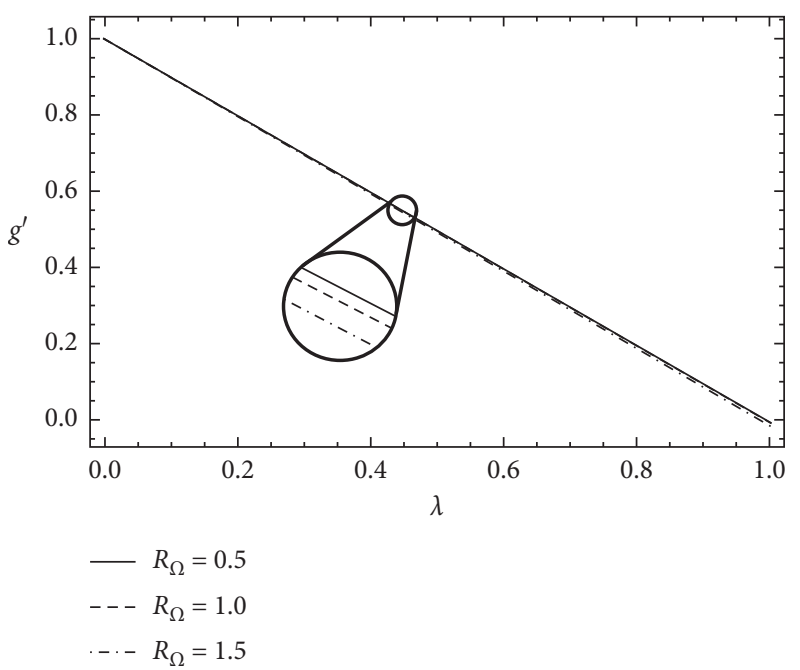

FIGURE 6: Effect of various values of rotational Reynolds number $R_{\Omega}$ on the tangential velocity distribution $g^{\prime}(\lambda)$.

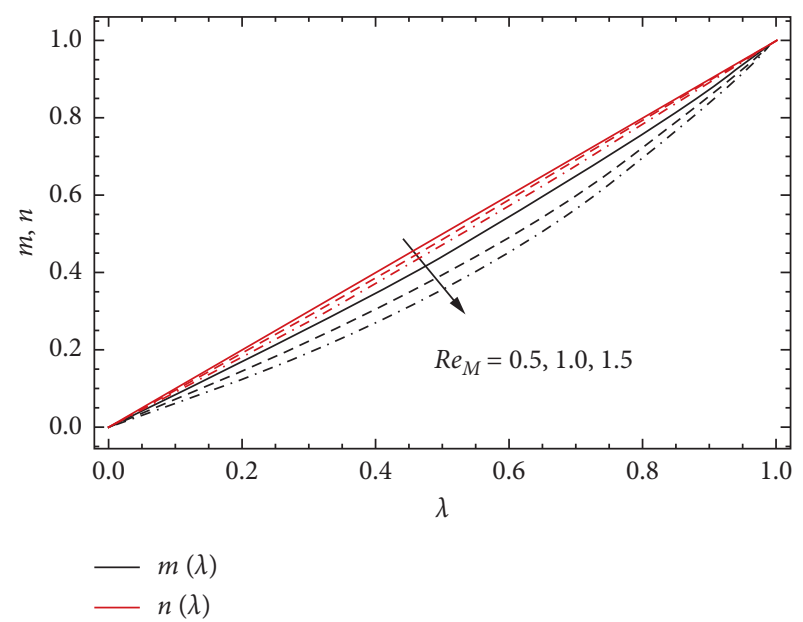

FIGURE 7: Effect of different values of magnetic Reynolds number $\mathrm{Re}_{M}$ on the axial and tangential induced magnetic field distributions $m(\lambda), n(\lambda)$.

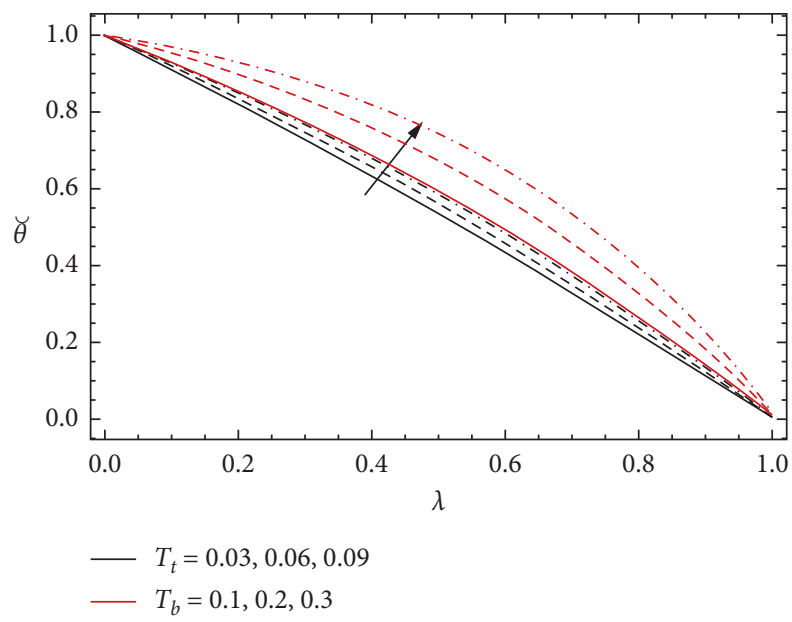

Figure 8: Effect of different values of thermophoresis parameter $T_{t}$ and Brownian motion parameter $T_{b}$ on the temperature distribution $\theta(\lambda)$.

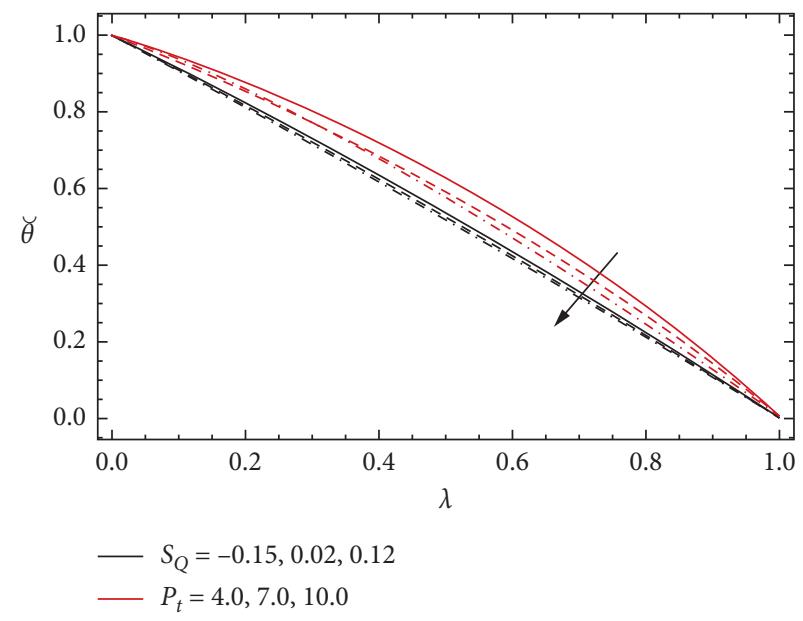

Figure 9: Effect of various values of squeeze Reynolds number $S_{Q}$ and Prandtl number $P_{t}$ on the temperature distribution $\theta(\lambda)$.

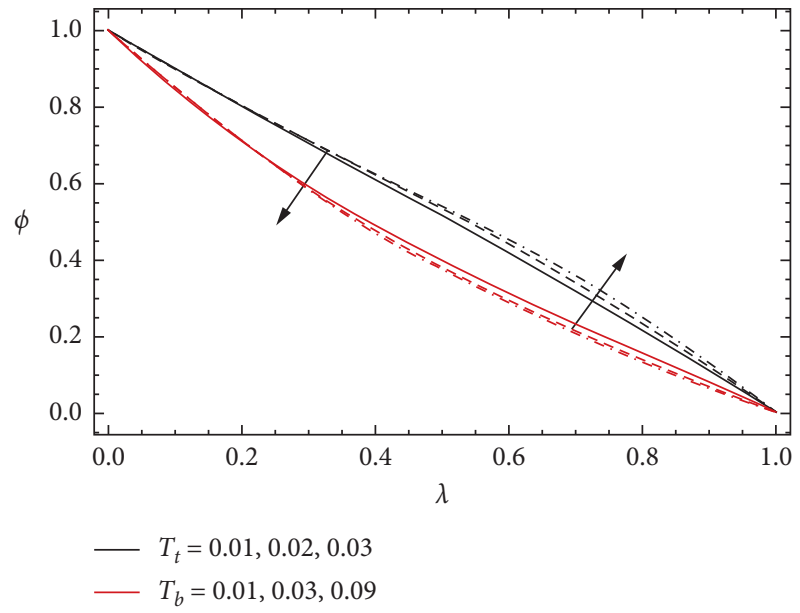

Figure 10: Effect of different values of thermophoresis parameter $T_{t}$ and Brownian motion parameter $T_{b}$ on the nanoparticle volume fraction $\phi(\lambda)$.

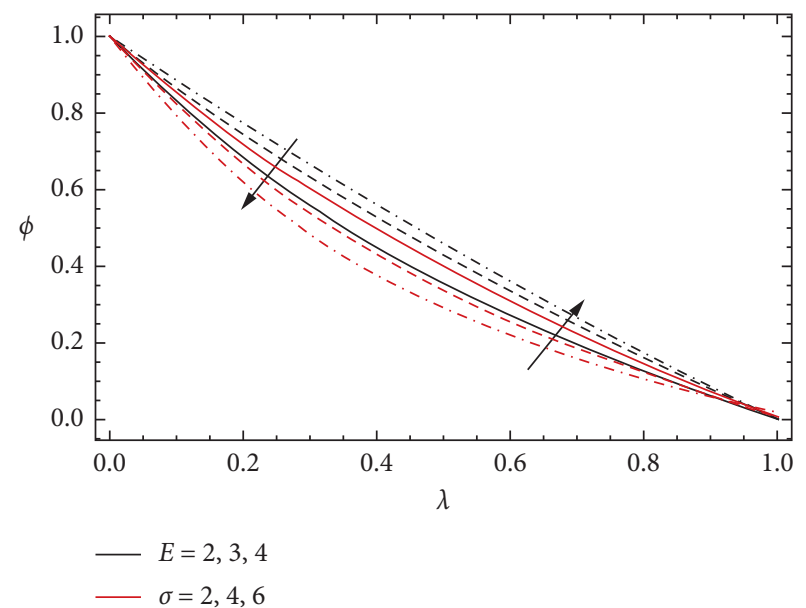

FIgURE 11: Effect of different values of activation energy $E$ and reaction rate $\sigma$ on the nanoparticle volume fraction $\phi(\lambda)$. 


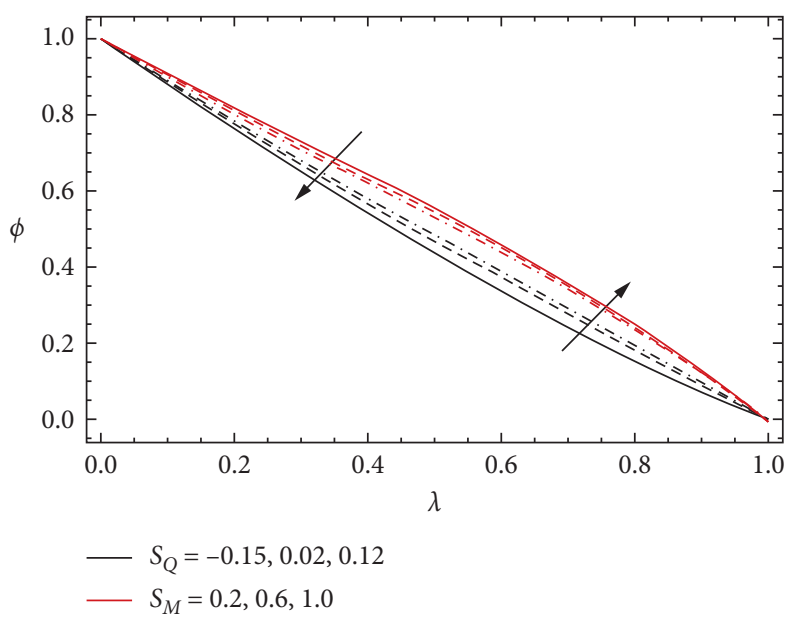

FIGURE 12: Effect of various values of squeeze Reynolds number $S_{Q}$ and Schmidt number $S_{M}$ on the nanoparticle volume fraction $\phi(\lambda)$.

that when increasing the value of the Prandtl number $P_{t}$, the thermal conductivity reduces, and due to this reason, the temperature profile declines. It is also seen that temperature profile decreases with an increase in the values of squeezed Reynolds number $S_{Q}$.

The impact of the thermophoresis parameter $T_{t}$ and the Brownian motion $T_{b}$ on the nanoparticle concentration $\phi(\lambda)$ is displayed in Figure 10. It is noticed that the concentration of nanoparticle declines by enhancing the Brownian motion parameter $T_{b}$ and concentration of nanoparticle enhances when values of the thermophoresis parameter $T_{t}$ increase. Figure 11 depicts the influence of activation energy $E$ and reaction rate $\sigma$ on the nanoparticle concentration $\phi(\lambda)$. It can be seen that, by enhancing the dimensionless activation energy $E$, the concentration of nanoparticle increases. That is because low temperatures and high energy activation contribute to a constant reaction rate, and thus, the chemical reaction slows down. As a result, the concentration of solute increases. Besides, with higher values of reaction rate $\sigma$, the nanoparticle concentration declines. Figure 12 shows the effect of squeezed Reynolds number $S_{Q}$ and Schmidt number $S_{M}$. By enhancing the values of squeezed Reynolds number $S_{Q}$, the nanoparticle concentration $\phi(\lambda)$ increases while the opposite behavior is observed when increasing the value of the Schmidt number $S_{M}$.

Figure 13 depicts the influence of squeezed Reynolds number $S_{Q}$ and Peclet number $P_{l}$ on microorganism density function $\chi(\lambda)$. It is perceived that, with the increase in the value of squeezed Reynolds number $S_{Q}$, the microorganism density function increases and it increases in the value of Peclet number $P_{l}$; the behavior of the microorganism density function shows the opposite phenomenon. The reason behind this is that the speed of microorganisms is decreased when there is a rise in the value of Peclet number, and therefore diffusivity of the microorganism decreases. Figure 14 demonstrates the physical behavior of the Schmidt number $S_{M}$. It is seen that the density of motile microorganism function increases by enhancing the value of the

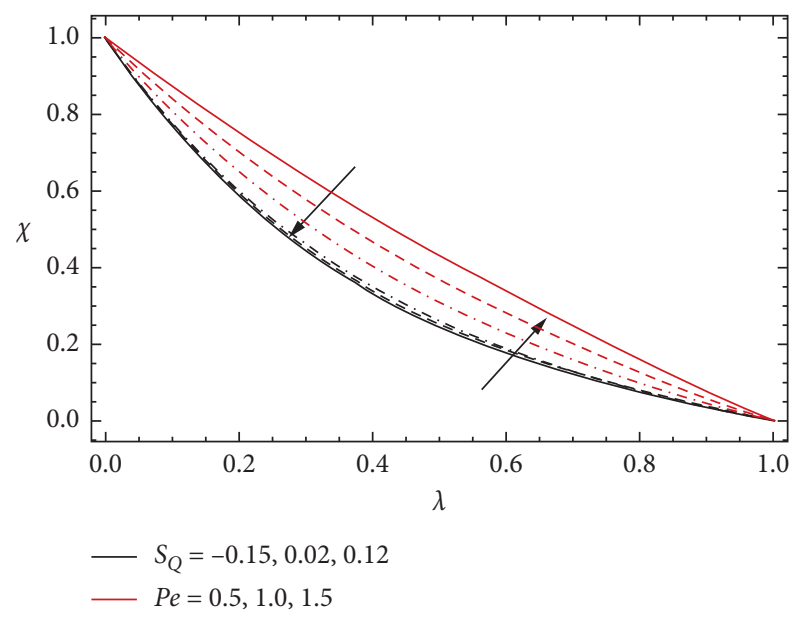

Figure 13: Effect of various values of squeeze Reynolds number $S_{Q}$ and Peclet number $\mathrm{Pe}$ on the motile microorganism density function $\chi(\lambda)$.

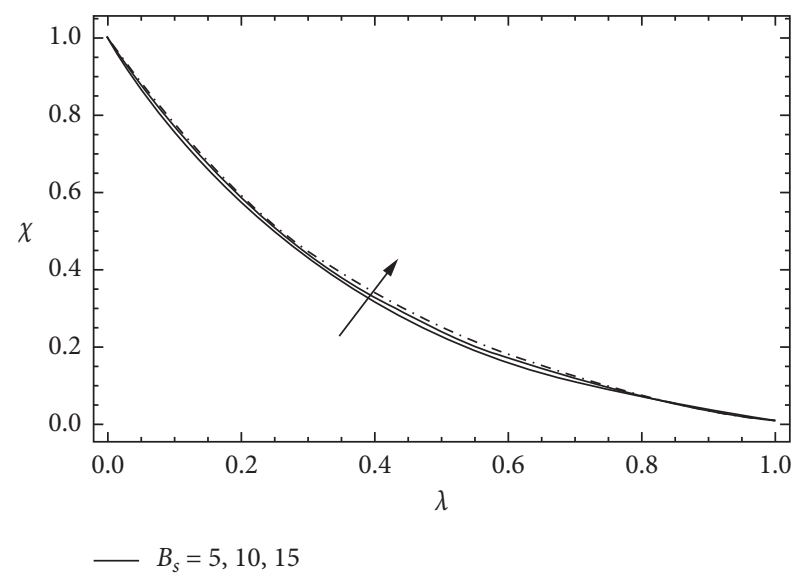

FIgURE 14: Effect of various values of bioconvection number $B_{s}$ on motile microorganism density function $\chi(\lambda)$.

bioconvection Schmidt number $B_{s}$, but the influence is nominal.

\section{Conclusion}

In this study, the flow behavior betwixt circular rotating plates filled with Carreau fluid under the suspension of nanoparticles and gyrotactic microorganisms in the presence of an induced magnetic field has been investigated. DTM-Padé is used to solve the resulting differential equations. Padé approximant is helpful to provide the swift convergence rate and provide stable results. It is observed that, after merging the differential transform method (DTM) with the Padé approximant, the results are far better, which is confirmed with the numerical shooting method. Tables are drawn for various values of flow parameters, and comparison is obtained with the shooting method, which shows that DTM-Padé is accurate and stable. The main findings are briefed as follows: 
(i) Rotational Reynolds number gives opposite behavior for the axial and tangential velocity distribution

(ii) The shear-thinning fluid viscosity decreases with shear strain due to its high velocity compared to Newtonian and shear-thickening fluids

(iii) In axial and tangential velocity distribution, velocity is decreasing by increasing the value of squeezed Reynolds number

(iv) The induced magnetic field in axial and tangential direction tends to reduce by enhancing the values of the magnetic Reynolds number

(v) In the temperature profile, by raising the Brownian motion and thermophoresis parameter, the temperature profile increases, but for nanoparticle concentration opposite effect has been observed

(vi) Squeezed Reynolds number suppresses the temperature profile, but opposite behavior is seen for concentration and microorganism profile

(vii) Activation energy enhances while the reaction rate suppresses the nanoparticle concentration

(viii) Peclet number reduces the microorganism profile, whereas squeezed Reynolds number and bioconvection number intend to enhance the microorganism profile

\section{Appendix}

The components form of Carreau fluid model are

$$
\begin{aligned}
\tau_{r r}=\mu_{0} & {\left[1+\frac{(n-1)}{2} \Gamma^{2}\left(2\left(\frac{\partial v_{r}}{\partial r}\right)^{2}+\left(\frac{\partial v_{r}}{\partial z}\right)^{2}+\left(\frac{\partial v_{\theta}}{\partial z}\right)^{2}+\left(\frac{\partial v_{\theta}}{\partial r}-\frac{v_{\theta}}{r}\right)^{2}+2\left(\frac{v_{r}}{r}\right)^{2}\right)\right] 2 \frac{\partial v_{r}}{\partial r}, } \\
\tau_{r \theta}= & \mu_{0}\left[1+\frac{(n-1)}{2} \Gamma^{2}\left(2\left(\frac{\partial v_{r}}{\partial r}\right)^{2}+\left(\frac{\partial v_{r}}{\partial z}\right)^{2}+\left(\frac{\partial v_{\theta}}{\partial z}\right)^{2}+\left(\frac{\partial v_{\theta}}{\partial r}-\frac{v_{\theta}}{r}\right)^{2}+2\left(\frac{v_{r}}{r}\right)^{2}\right)\right]\left(\frac{\partial v_{\theta}}{\partial r}-\frac{v_{\theta}}{r}\right), \\
\tau_{r z}=\mu_{0} & {\left[1+\frac{(n-1)}{2} \Gamma^{2}\left(2\left(\frac{\partial v_{r}}{\partial r}\right)^{2}+\left(\frac{\partial v_{r}}{\partial z}\right)^{2}+\left(\frac{\partial v_{\theta}}{\partial z}\right)^{2}+\left(\frac{\partial v_{\theta}}{\partial r}-\frac{v_{\theta}}{r}\right)^{2}+2\left(\frac{v_{r}}{r}\right)^{2}\right)\right] \frac{\partial v_{r}}{\partial z}, } \\
\tau_{\theta r}=\mu_{0} & {\left[1+\frac{(n-1)}{2} \Gamma^{2}\left(2\left(\frac{\partial v_{r}}{\partial r}\right)^{2}+\left(\frac{\partial v_{r}}{\partial z}\right)^{2}+\left(\frac{\partial v_{\theta}}{\partial z}\right)^{2}+\left(\frac{\partial v_{\theta}}{\partial r}-\frac{v_{\theta}}{r}\right)^{2}+2\left(\frac{v_{r}}{r}\right)^{2}\right)\right]\left(\frac{\partial v_{\theta}}{\partial r}-\frac{v_{\theta}}{r}\right), } \\
\tau_{\theta \theta}= & \mu_{0}\left[1+\frac{(n-1)}{2} \Gamma^{2}\left(2\left(\frac{\partial v_{r}}{\partial r}\right)^{2}+\left(\frac{\partial v_{r}}{\partial z}\right)^{2}+\left(\frac{\partial v_{\theta}}{\partial z}\right)^{2}+\left(\frac{\partial v_{\theta}}{\partial r}-\frac{v_{\theta}}{r}\right)^{2}+2\left(\frac{v_{r}}{r}\right)^{2}\right)\right] 2\left(\frac{v_{r}}{r}\right), \\
\tau_{z \theta}=\mu_{0} & {\left[1+\frac{(n-1)}{2} \Gamma^{2}\left(2\left(\frac{\partial v_{r}}{\partial r}\right)^{2}+\left(\frac{\partial v_{r}}{\partial z}\right)^{2}+\left(\frac{\partial v_{\theta}}{\partial z}\right)^{2}+\left(\frac{\partial v_{\theta}}{\partial r}-\frac{v_{\theta}}{r}\right)^{2}+2\left(\frac{v_{r}}{r}\right)^{2}\right)\right] \frac{\partial v_{\theta}}{\partial z}, } \\
\tau_{z z}= & 0 . \\
\tau_{z r}=\mu_{0} & {\left[1+\frac{(n-1)}{\partial r} \Gamma^{2}\left(2\left(\frac{\partial v_{r}}{\partial z}\right)^{2}+\left(\frac{\partial v_{r}}{\partial z}\right)^{2}+\left(\frac{\partial v_{\theta}}{\partial z}\right)^{2}+\left(\frac{\partial v_{\theta}}{\partial r}-\frac{v_{\theta}}{r}\right)^{2}+2\left(\frac{v_{r}}{r}\right)^{2}\right)\right] \frac{\partial v_{\theta}}{\partial z}, } \\
\left.\left.\tau_{\theta z}=\mu_{0}\left[1+\frac{\partial v_{\theta}}{\partial z}\right)^{2}+\left(\frac{\partial v_{\theta}}{\partial r}-\frac{v_{\theta}}{r}\right)^{2}+2\left(\frac{v_{r}}{r}\right)^{2}\right)\right] \frac{\partial v_{r}}{\partial z}, &
\end{aligned}
$$




\section{Data Availability}

No data were used to support this study.

\section{Conflicts of Interest}

The authors declare that they have no conflicts of interest.

\section{Acknowledgments}

M. M. Bhatti was supported by the Cultivation Project of Young and Innovative Talents in Universities of Shandong Province (Nonlinear Sciences Research Team).

\section{References}

[1] R. Bird, R. Byron, R. C. Armstrong, and O. Hassager, Dynamics of Polymeric Liquids. Vol. 1: Fluid Mechanics, Wiley, London, UK, 1987.

[2] P. J. Carreau, "Rheological equations from molecular network theories," Transactions of the Society of Rheology, vol. 16, no. 1, pp. 99-127, 1972.

[3] R. I. Yahaya, N. Md Arifin, and S. S. P. Mohamed Isa, "Stability analysis of MHD Carreau fluid flow over a permeable shrinking sheet with thermal radiation," Sains Malaysiana, vol. 48, no. 10, pp. 2285-2295, 2019.

[4] M. R. Eid, K. L. Mahny, A. Dar, and T. Muhammad, "Numerical study for carreau nanofluid flow over a convectively heated nonlinear stretching surface with chemically reactive species," Physica A: Statistical Mechanics and Its Applications, vol. 540, Article ID 123063, 2020.

[5] P. N. Santoshi, G. V. R. Reddy, and P. Padma, "Numerical study of Carreau nanofluid flow under slips," International Journal of Applied and Computational Mathematics, vol. 5, no. 5 , p. $122,2019$.

[6] M. Khan, T. Salahuddin, M. Y. Malik, and F. Khan, "Change in internal energy of Carreau fluid flow along with Ohmic heating: a Von Karman application," Physica A: Statistical Mechanics and Its Applications, vol. 547, Article ID 123440, 2020.

[7] S. Bilal, A. S. Shafqatullah, A. S. Alshomrani et al., "Analysis of Carreau fluid in the presence of thermal stratification and magnetic field effect," Results in Physics, vol. 10, pp. 118-125, 2018.

[8] M. Khan, M. Azam, and A. S. Alshomrani, "On unsteady heat and mass transfer in Carreau nanofluid flow over expanding or contracting cylinder with convective surface conditions," Journal of Molecular Liquids, vol. 231, pp. 474-484, 2017.

[9] N. S. Khan, S. Islam, T. Gul, I. Khan, W. Khan, and L. Ali, "Thin film flow of a second grade fluid in a porous medium past a stretching sheet with heat transfer," Alexandria Engineering Journal, vol. 57, no. 2, pp. 1019-1031, 2018.

[10] A. Shafiq, T. N. Sindhu, and C. M. Khalique, "Numerical investigation and sensitivity analysis on bioconvective tangent hyperbolic nanofluid flow towards stretching surface by response surface methodology," Alexandria Engineering Journal, 2020.

[11] A. Shafiq, Z. Hammouch, T. Naz Sindhu, and D. Baleanu, "Statistical approach of mixed convective flow of third-grade fluid towards an exponentially stretching surface with convective boundary condition," Special Functions and Analysis of Differential Equations, p. 307, 2020.

[12] N. Acharya, K. Das, and P. K. Kundu, "The squeezing flow of $\mathrm{Cu}$-water and $\mathrm{Cu}$-kerosene nanofluids between two parallel plates," Alexandria Engineering Journal, vol. 55, no. 2, pp. 1177-1186, 2016.

[13] N. S. Khan, T. Gul, M. A. Khan, E. Bonyah, and S. Islam, "Mixed convection in gravity-driven thin film non-Newtonian nanofluids flow with gyrotactic microorganisms," Results in Physics, vol. 7, pp. 4033-4049, 2017.

[14] D. Lu, M. Ramzan, N. ul Huda, J. D. Chung, and U. Farooq, "Nonlinear radiation effect on MHD Carreau nanofluid flow over a radially stretching surface with zero mass flux at the surface," Scientific Reports, vol. 8, no. 1, pp. 1-17, 2018.

[15] C. H. Amanulla, A. Wakif, Z. Boulahia, M. S. Reddy, and N. Nagendra, "Numerical investigations on magnetic field modeling for Carreau non-Newtonian fluid flow past an isothermal sphere," Journal of the Brazilian Society of Mechanical Sciences and Engineering, vol. 40, no. 9, p. 462, 2018.

[16] I. Khan, M. Shafquatullah, M. Y. Malik, A. Hussain, and M. Khan, "Magnetohydrodynamics Carreau nanofluid flow over an inclined convective heated stretching cylinder with Joule heating," Results in Physics, vol. 7, pp. 4001-4012, 2017.

[17] I. Khan, S. Ullah, M. Y. Malik, and A. Hussain, "Numerical analysis of MHD Carreau fluid flow over a stretching cylinder with homogenous-heterogeneous reactions," Results in Physics, vol. 9, pp. 1141-1147, 2018.

[18] H. Sardar, M. Khan, and L. Ahmad, "Local non-similar solutions of convective flow of Carreau fluid in the presence of MHD and radiative heat transfer," Journal of the Brazilian Society of Mechanical Sciences and Engineering, vol. 41, no. 2, p. 69, 2019.

[19] T. Salahuddin, A. Hussain, M. Y. Malik, M. Awais, and M. Khan, "Carreau nanofluid impinging over a stretching cylinder with generalized slip effects: using finite difference scheme," Results in Physics, vol. 7, pp. 3090-3099, 2017.

[20] M. M. Bhatti, R. Ellahi, A. Zeeshan, M. Marin, and N. Ijaz, "Numerical study of heat transfer and Hall current impact on peristaltic propulsion of particle-fluid suspension with compliant wall properties," Modern Physics Letters B, vol. 33, no. 35, Article ID 1950439, 2019.

[21] M. Bovand, S. Rashidi, J. A. Esfahani, S. C. Saha, Y. T. Gu, and M. Dehesht, "Control of flow around a circular cylinder wrapped with a porous layer by magnetohydrodynamic," Journal of Magnetism and Magnetic Materials, vol. 401, pp. 1078-1087, 2016.

[22] N. G. Rudraswamy, S. A. Shehzad, K. Ganesh Kumar, and B. J. Gireesha, "Numerical analysis of MHD three-dimensional Carreau nanoliquid flow over bidirectionally moving surface," Journal of the Brazilian Society of Mechanical Sciences and Engineering, vol. 39, no. 12, pp. 5037-5047, 2017.

[23] K. A. Kumar, J. V. R. Reddy, V. Sugunamma, and N. Sandeep, "MHD Carreau fluid flow past a melting surface with cattaneo-christov heat flux," in Proceedings of the Applied Mathematics and Scientific Computing, pp. 325-336, Vellore, India, December 2019.

[24] N. S. Akbar, S. Nadeem, R. U. Haq, and S. Ye, "MHD stagnation point flow of Carreau fluid toward a permeable shrinking sheet: dual solutions," Ain Shams Engineering Journal, vol. 5, no. 4, pp. 1233-1239, 2014.

[25] G. C. Shit and S. Mukherjee, "MHD graphene-polydimethylsiloxane Maxwell nanofluid flow in a squeezing channel with thermal radiation effects," Applied Mathematics and Mechanics, vol. 40, no. 9, pp. 1269-1284, 2019.

[26] A. R. Bestman, "Radiative heat transfer to flow of a combustible mixture in a vertical pipe," International Journal of Energy Research, vol. 15, no. 3, pp. 179-184, 1991. 
[27] S. Anuradha and M. Yegammai, "MHD radiative boundary layer flow of nanofluid past a vertical plate with effects of binary chemical reaction and activation energy," The Global Journal of Pure and Applied Mathematics, vol. 13, pp. 6377-6392, 2017.

[28] M. Irfan, W. A. Khan, M. Khan, and M. M. Gulzar, "Influence of Arrhenius activation energy in chemically reactive radiative flow of 3D Carreau nanofluid with nonlinear mixed convection," Journal of Physics and Chemistry of Solids, vol. 125, pp. 141-152, 2019.

[29] M. I. Khan, M. Irfan, W. A. Khan, M. Waqas, and S. Rashi, "Activation energy analysis in entropy optimized reactive flow," Applied Nanoscience, vol. 10, pp. 2673-2683, 2020.

[30] K. G. Kumar, A. Baslem, B. C. Prasannakumara, J. Majdoubi, M. Rahimi-Gorji, and S. Nadeem, "Significance of Arrhenius activation energy in flow and heat transfer of tangent hyperbolic fluid with zero mass flux condition," Microsystem Technologies, vol. 26, pp. 2517-2526, 2020.

[31] M. K. Nayak, J. Prakash, D. Tripathi, V. S. Pandey, S. Shaw, and O. D. Makinde, "3D Bioconvective multiple slip flow of chemically reactive Casson nanofluid with gyrotactic microorganisms," Heat Transfer-Asian Research, vol. 49, no. 1, pp. 135-153, 2020.

[32] I. Tlili, H. Waqas, A. Almaneea, S. U. Khan, and M. Imran, "Activation energy and second order slip in bioconvection of Oldroyd-B nanofluid over a stretching cylinder: a proposed mathematical model," Processes, vol. 7, no. 12, p. 914, 2019.

[33] Z. Abdelmalek, S. Ullah Khan, H. Waqas, H. A. Nabwey, and I. Tlili, "Utilization of second order slip, activation energy and viscous dissipation consequences in thermally developed flow of third grade nanofluid with gyrotactic microorganisms," Symmetry, vol. 12, no. 2, p. 309, 2020.

[34] S. M. Atif, S. Hussain, and M. Sagheer, "Magnetohydrodynamic stratified bioconvective flow of micropolar nanofluid due to gyrotactic microorganisms," AIP Advances, vol. 9, no. 2, Article ID 25208, 2019.

[35] M. I. Khan, F. Haq, T. Hayat, A. Alsaedi, and M. U. Rahman, "Natural bio-convective flow of Sisko nanofluid subject to gyrotactic microorganisms and activation energy," Physica Scripta, vol. 94, no. 12, Article ID 125203, 2019.

[36] M. M. Bhatti, A. Shahid, T. Abbas, S. Z. Alamri, and R. Ellahi, "Study of activation energy on the movement of gyrotactic microorganism in a magnetized nanofluids past a porous plate," Processes, vol. 8, no. 3, p. 328, 2020.

[37] V. Nagendramma, C. S. K. Raju, B. Mallikarjuna, S. A. Shehzad, and A. Leelarathnam, "3D Casson nanofluid flow over slendering surface in a suspension of gyrotactic microorganisms with Cattaneo-Christov heat flux," Applied Mathematics and Mechanics, vol. 39, no. 5, pp. 623-638, 2018.

[38] M. I. Khan, F. Alzahrani, A. Hobiny, and Z. Ali, "Estimation of entropy optimization in Darcy-Forchheimer flow of CarreauYasuda fluid (non-Newtonian) with first order velocity slip," Alexandria Engineering Journal, vol. 59, no. 5, pp. 3953-3962, 2020.

[39] R. Muhammad, M. I. Khan, M. Jameel, and N. B. Khan, "Fully developed Darcy-Forchheimer mixed convective flow over a curved surface with activation energy and entropy generation," Computer Methods and Programs in Biomedicine, vol. 188, Article ID 105298, 2020.

[40] M. I. Khan and F. Alzahrani, "Binary chemical reaction with activation energy in dissipative flow of non-Newtonian nanomaterial," Journal of Theoretical and Computational Chemistry, vol. 19, Article ID 2040006, 2020.

[41] Z. Shah, E. Alzahrani, M. Jawad, and U. Khan, "Microstructure and inertial characteristics of MHD suspended
SWCNTs and MWCNTs based maxwell nanofluid flow with bio-convection and entropy generation past A permeable vertical cone," Coatings, vol. 10, no. 10, p. 998, 2020.

[42] A. Khan, Z. Shah, E. Alzahrani, and S. Islam, "Entropy generation and thermal analysis for rotary motion of hydromagnetic Casson nanofluid past a rotating cylinder with Joule heating effect," International Communications in Heat and Mass Transfer, vol. 119, Article ID 104979, 2020.

[43] P. T. Griffiths, "Flow of a generalised Newtonian fluid due to a rotating disk," Journal of Non-newtonian Fluid Mechanics, vol. 221, pp. 9-17, 2015.

[44] M. M. Rashidi, N. Freidoonimehr, E. Momoniat, and B. Rostami, "Study of nonlinear MHD tribological squeeze film at generalized magnetic Reynolds numbers using DTM," PloS One, vol. 10, no. 8, Article ID e0135004, 2015.

[45] S. S. Elshekh, M. K. Abd Elhady, and F. N. Ibrahim, "Fluid film squeezed between two rotating disks in the presence of a magnetic field," International Journal of Engineering Science, vol. 34, no. 10, pp. 1183-1195, 1996.

[46] R. Abazari and A. Borhanifar, "Numerical study of the solution of the Burgers and coupled Burgers equations by a differential transformation method," Computers \& Mathematics with Applications, vol. 59, no. 8, pp. 2711-2722, 2010.

[47] M. M. Rashidi, O. A. Beg, M. Asadi, and M. T. Rastegari, "DTM-Padé modeling of natural convective boundary layer flow of a nanofluid past a vertical surface," International Journal of Thermal \& Environmental Engineering, vol. 4, no. 1, pp. 13-24, 2012.

[48] M. M. Bhatti, S. Ullah Khan, O. Anwar Bég, and A. Kadir, "Differential transform solution for Hall and ion-slip effects on radiative-convective Casson flow from a stretching sheet with convective heating," Heat Transfer, vol. 49, no. 2, pp. 872-888, 2020 Mar.

[49] L. Zhang, M. B. Arain, M. M. Bhatti, A. Zeeshan, and H. HalSulami, "Effects of magnetic Reynolds number on swimming of gyrotactic microorganisms between rotating circular plates filled with nanofluids," Applied Mathematics and Mechanics, vol. 41, no. 4, pp. 637-654, 2020.

[50] T. Y. Na, Computational Methods in Engineering Boundary Value Problems, Vol. 145, Academic Press, Cambridge, MA, USA, 1980. 